Éric AuNOBLE

Université de Genève

\title{
La Pologne, un sujet soviétique ? Représentations filmiques et trajectoires personnelles, 1939-1945
}

"Spoliations » et « transferts »: les deux termes semblent particulièrement s'appliquer aux relations soviéto-polonaises pendant la Seconde Guerre mondiale. «Spoliation » de toute la partie Est de la Pologne au profit de l'Union soviétique aboutissant, conjointement avec l'invasion nazie, à la disparition du pays en vertu du pacte Molotov-Ribbentrop. «Transfert » massif de population, un million de citoyens polonais ayant été déportés à l'intérieur du territoire soviétique (Gross 2002 : 194). Si la Pologne apparait clairement en position de victime, elle exerce tout de même une influence paradoxale sur l'URSS. Elle oblige le géant soviétique à se justifier, à présenter l'annexion de la Pologne orientale comme " La libération des terres ukrainiennes et biélorusses du joug des seigneurs polonais et la réunification des peuples frères dans une famille unie », (Dovženko 1940). Malgré la répression dont ils font l'objet, les ci-devant Polonais déportés en URSS doivent être reconnus par un statut spécifique, ce qui nécessite de compliqués montages juridiques (Gousseff 2015). On ne saurait ignorer non plus que la déportation soviétique sauva finalement

1 Un première version de ce travail a été présentée aux journées d'étude « Circulations intellectuelles en temps de guerre - Espace européen et Union soviétique (1939-1945) » (Paris 8, 17-18.06.2015), coorganisées par Rachel Mazuy (IHTP), Marianne MatardBonucci (Paris 8), Marie-Cécile Bouju (Paris 8) et Isabelle Gouarné (Curapp/ CNRS). Qu'elles soient vivement remerciées de l'occasion qu'elles m'ont donnée d'aborder ce sujet. 
de l'extermination nazie les 300000 Juifs parmi les citoyens polonais déplacés (Gross $2002: 269)$.

Pour paraphraser l'écrivain Vladimir Sorokine ${ }^{2}$, c'est en fait la Pologne qui était entrée en URSS en 1939. La spécificité soviétique tant au niveau institutionnel qu'idéologique dans le concert des nations fait l'originalité de ce processus. Les tentatives de la grande puissance pour intégrer la Pologne traduisent surtout une difficulté à la digérer, d'autant que de 1939 à 1945 la conjoncture change et avec elle les représentations. En 1940, le film Libération évoque un «État polonais artificiel » qui s'effondre au bout de dix jours de guerre (Dovženko 1940 : 4'33") alors qu'en 1945, le carton qui ouvre Zigmund Kolosovski affirme : «Quand les Allemands - ennemis éternels des Slaves - ont jeté leurs unités blindées sur la Pologne, ont commencé pour le peuple polonais les heures les plus sombres de son histoire " (Navrockij $1945: 1$ '36"). On voit que le cinéma est au premier rang des moyens de diffusion du discours officiel, tout en participant à la production des représentations. Après avoir étudié dans un précédent travail le rôle du cinéma d'actualité et documentaire dans ce processus (Aunoble 2014), je m'intéresserai ici au cinéma de fiction.

Il s'agira d'étudier l'image - évolutive - de la Pologne fabriquée à l'usage des Soviétiques. Au-delà de son évidente instrumentalisation dans l'appareil de propagande soviétique, on soulignera ici les échanges soviéto-polonais réels (même s'ils sont contraints) qui permettent la formation de cette image et on s'interrogera donc particulièrement sur les personnes qui incarnent ces transferts idéologiques et culturels en travaillant à l'élaboration de ces films : réalisateurs, scénaristes et acteurs, eux-mêmes pris dans cette histoire qu'ils représentent. Parmi eux, la figure de Wanda Wasilewska s'impose. Polonaise si bien ralliée aux Soviétiques qu'elle finira ses jours en URSS, elle participe, à côté de sa carrière politique, à la vie culturelle de la période, alternativement comme Polonaise ou comme Soviétique.

\section{La Pologne, une fiction cinématographique}

L'annexion de régions de Pologne par l'URSS apparaît du point de vue soviétique comme une revanche sur l'histoire et une partie de la filmographie reviendra sur le contentieux séculaire polono-russe où l'Ukraine et la Biélorussie font figure d'enjeux territoriaux : quand Années de feu (Ognennye gody) de Vladimir KorchSabline (Korš-Sablin 1939) rappelle le moment de la guerre civile en 1920 quand les Rouges s'opposèrent aux Biélopoliaki (« Polonais blancs »), Bogdan Khmelnitski d'Igor Savtchenko (Savčenko 1941) retrace la grande révolte des cosaques ukrainiens

2 Vladimir Sorokine évoque l'annexion de la Crimée par la Russie en disant «L'Ukraine est entrée en nous » (Sorokin 2014). 
contre la szląchta, la noblesse polonaise, au XVII ${ }^{e}$ siècle (Yekelchyk 2004). Face à la réactivation de cette mémoire clivante, on ne voit pas à l'écran de promotion d'une polonité positive, progressiste dans le sens soviétique. Ainsi, le projet d'un film sur Adam Mickewicz, pourtant «grand poète polonais [qui] a connu des périodes grandioses d'inspiration révolutionnaire $»^{3}$, n'est jamais réalisé (Tokarev 2017).

Non content de rejouer les conflits anciens, le cinéma soviétique de fiction met également en scène les événements les plus récents. L'annexion de l'Est de la Pologne est montrée par le regard de kolkhoziens biélorusses - qui s'en réjouissent évidemment - dans La Famille Ianouch (Navrockij 1941). Deux films adoptent au contraire un point de vue interne en suivant un groupe de ressortissants polonais de l'immédiat avant-guerre jusqu'à l'arrivée des Soviétiques en 1939: Vent d'Est (Veter s Vostoka) d'Abram Room (Room 1940) et Le Rêve de Mikhaïl Romm (Romm 1941). Dans un contexte bien différent, à la fin de la guerre, Zigmund Kolosovski (Navrockij 1945) évoque la résistance polonaise face à l'occupant nazi. Ce corpus est disparate. Si Le Rêve est un chef d'œuvre que son auteur considéra comme un de ses films les plus personnels, Vent d'Est est assez ambitieux mais très inégal alors que Zigmund Kolosovski est un film d'aventure construit comme la succession des exploits du héros. Zigmund Kolosovski atteindra le sommet du box office soviétique en 1946 (18,2 millions de spectateurs), alors que Le Rêve ne fut distribué qu'à partir de 1943 et avec parcimonie (Romm 1981 : 331, 390 ; Anotovanyj... $2011:$ 142). Et inutile de dire que la Pologne des films de 1940-1941 n'a rien à voir avec celle présentée en 1945. Dans tous les cas pourtant, il s'agit d'une Pologne de fiction construite pour les Soviétiques.

Les films de 1940-1941 visent à dénoncer le système social qui régnait en Pologne avant la « libération » par les Soviétiques. Par exemple, Mikhaïl Romm devait illustrer « la réunification des Biélorusses de l'ouest avec leurs frères de l'est. Le destin de la paysannerie biélorusse dans la Pologne féodale. Le joug des grands propriétaires, des capitalistes et des fonctionnaires sur fond de paupérisation des masses laborieuses » (Astangov 1971 : 230). L'action ayant été déplacée de Biélorussie en Ukraine, le film de Mikhail Romm forme avec celui d'Abram Room un diptyque illustrant cette situation car Vent d'Est décrit la campagne et Le Rêve, la ville. Vent d'Est retrace les vicissitudes d'un paysan, Khoma Gabrys, en butte à la spoliation de la part de la comtesse Przeżyńska : elle le prive de son dernier lopin de terre pour percer une route. Comme il récupère symboliquement quelques sacs de «sa terre », il est condamné à une amende qui le transforme en serf sous le contrôle de l'intendant du domaine, un homme sans pitié. Après que la « Justice » polonaise l'a condamné, la police de ce même État assassine sa femme qui résistait à la saisie de leurs biens. Ganna, la jeune

3 Archives centrales du musée de la littérature et des arts d'Ukraine (CDAMLM, Kiev), f. 670, op. 2, d. 9 : Aleksej Spešnev, Adam Mickevič, Literaturnyj scenarij, 1940, 1. 5 a. 
institutrice nommée dans le village à sa sortie du couvent, assiste impuissante aux drames, alors même qu'on l'empêche d'instruire convenablement les petits paysans.

Comme une suite de Vent d'Est, le second film, Le Rêve, suit le destin d'Anna, une jeune paysanne obligée de quitter son village car sa famille est trop pauvre. Elle part gagner sa vie dans une ville qui compte « Cinq cathédrales, deux prisons, quatre usines, trente-neuf maisons de tolérance et deux-cent-quarante boutiques » (Romm 1941 : 3'47'-3'55"). La pension de famille "Le Rêve » où elle s'installe est le condensé de cette société décadente. «Le Rêve » est tenu d'une main de fer par Róża Skorochod, qui après des années passées à faire des ménages, a réussi à devenir commerçante et à payer des études à son fils, Lazare. Malgré son diplôme d'ingénieur, ce dernier est au chômage. Il passe donc son temps à la pension, participant aux zizanies qui occupent les résidents : un tailleur et un peintre vieillissants, un cocher au bord de la ruine et une trentenaire cherchant désespérément un mari. Celle-ci finira par se suicider en découvrant que son promis trouvé par petite annonce, un homme qui présentait si bien et lui avait promis monts et merveilles, est en fait un coureur de dot qui vit dans une soupente en nettoyant de façon maniaque son unique costume.

Ces films reflètent une certaine réalité. La région de Lwów ${ }^{4}$ dans la République polonaise (comme auparavant la Galicie orientale dans l'empire austro-hongrois) était traversée de clivages indissociablement sociaux et ethniques : à des villes polonojuives s'opposent des campagnes largement ukrainiennes ${ }^{5}$; dans les premières, peu industrialisées, vivent des propriétaires terriens héritiers de la noblesse et des fonctionnaires ainsi qu'une petite bourgeoisie souvent juive ; les paysans forment la base d'une économie encore arriérée et leur travail, contrôlé par des intendants, crée la richesse dont le reste de la société dispose. On remarquera d'abord que la composante juive de la population est absente de Vent d'Est. Sa présence dans Le Rêve est ambiguë : il est évident pour un spectateur soviétique que Róża Skorochod et son fils Lazare sont juifs, mais cela n'est jamais précisé explicitement. Néanmoins, leur situation sociale dans une petite-bourgeoisie dans la gêne est assez bien décrite. Surtout, leur statut discriminé est admirablement rendu dans une simple réplique. La célibataire lit son annonce matrimoniale à la logeuse : «Jeune fille sachant tenir un ménage recherche union solide et aisée. Veufs chargés de famille et Juifs s'abstenir ». Róża Skorochod commente : « Oui, les Juifs s'abstiendront. Lisez la suite ». (Romm

4 Lemberg (all.), Lwów (pol.), Lvov (rus.), Lviv (ukr.). Dans cet article, nous employons à chaque fois la graphie du locuteur et/ou du moment de référence.

5 À Lwów, $50 \%$ de « catholiques-romains » (Polonais), $32 \%$ d' « israélites » (Juifs) et $16 \%$ de « gréco-catholiques » (Ukrainiens). Dans le reste de la région (voïévodie), la proportion de « catholiques-romains » tombe à $45 \%$, celle des « israélites » à $8,6 \%$ alors que les « gréco-catholiques » représentent $44 \%$ des habitants ; mais à l'est de la région, ils sont très majoritaires (Statystyka Polski 1937-1938). 
$1941: 15$ '28"). Stigmate de la Pologne d'avant-guerre mais aussi phénomène présent en URSS, l'antisémitisme est ainsi représenté et dénoncé à l'écran fut-ce avec quelques précautions. Le fait est assez rare en 1940-1941 pour être souligné (Hicks 2012 : 42).

Ces films ne se réduisent pas au simple placage de la grille communiste de la lutte des classes sur la réalité polonaise. D'ailleurs, même quand elles mettent bien en scène l'affrontement entre des groupes sociaux constitués, ces deux œuvres ne ressortent pas vraiment du marxisme. On notera d'abord que le modèle présenté au spectateur soviétique est le paysan ukrainien et non l'ouvrier sans patrie. Assez logiquement absent du drame rural Vent d'Est, le prolétariat apparaît dans Le Rêve avec le visage du frère d'Anna, qui travaille à l'usine; mais il ne joue pas de rôle essentiel. De l'autre côté, l'ennemi est moins caractérisé par sa situation socio-économique que par sa culture. Le château de la comtesse Przeżyńska constitue un décor oppressant de salles hautes et grandioses au mobilier sombre et massif [fig. 01], aussi exotique pour un Soviétique que la ville de Lvov, le décor (partiellement reconstitué) du Rêve. Le cadre urbain, souvent filmé de nuit, donne également une impression de noirceur, régulièrement relevée par les enseignes et les publicités qui dénotent le consumérisme [fig. 02]. La modernité, à la fois dans son caractère européen et capitaliste, est ainsi connotée négativement. Enfin, l'oppression est marquée par les symboles religieux catholiques (statue de la vierge et crucifix) qui confondent en un tout la confession majoritaire des Polonais (catholique-romaine) et celle des Ukrainiens (grécocatholique) [fig. 03].

On assiste ainsi à un double glissement idéologique : de la centralité ouvrière vers le primat paysan et du discours de classe vers l'analyse ethno-culturelle. Loin du marxisme, on est plutôt dans la veine populiste du XIX siècle, et même pas dans son acception révolutionnaire russe (narodničestvo), mais plutôt dans celle des proto-nationalistes ukrainiens (narodovstvo) (Himka $1983: 45$ ). Vent d'Est donne à voir les costumes folkloriques ukrainiens [fig. 04] et consacre une longue scène aux danses d'une fête villageoise (Room $1940: 24$ '25"'28'57'). Tourné aux confins de la Hongrie, le film s'inscrit bon gré mal gré dans le topos nationaliste qui voit dans les Carpates le berceau de la nation ukrainienne (Magocsi 1978 : 123 et ss.). Plus qu'un ferment révolutionnaire collectiviste, le paysan ukrainien apparaît comme le réceptacle de l'authenticité nationale face à l'acculturation promue par le pouvoir polonais. Décrivant ainsi dans une perspective nationale le sort d'une minorité opprimée à laquelle le " grand frère » extérieur viendra finalement en aide, Vent d'Est est comparable de ce point de vue à Retour au pays (Gustav Ucicky, Heimkehr, Potsdam : Universum Film AG, 1941), le film allemand qui justifie l'annexion de la Pologne occidentale par le sort des germanophones sous la Rzeczpospolita (Deak 2011 ; Dufour 2015).

Si les paysans ne font pas la révolution et peinent même à résister à l'oppression seigneuriale, on a vu dans Le Rêve qu'il ne fallait pas attendre grand-chose de la 
population urbaine. Les contestataires ne représentent pas plus un espoir. Des personnages de communistes, de "bolcheviks », figurent évidemment comme héros positifs dans les deux films ${ }^{6}$. Néanmoins ils subissent la répression, ils sont pourchassés et arrêtés par la police polonaise. Même si leur militantisme amène la prise de conscience des héroïnes qui en tombent toutes deux amoureuses [fig. 05], le destin des protagonistes est globalement un constat d'échec. L'institutrice Ganna, qui symbolise l'intelligentsia nationale dans Vent d'Est, est réduite à l'impuissance, on l'a dit. Chez Mikhail Romm, Anna, la jeune paysanne partie chercher fortune en ville, perd finalement son travail et son logis. Elle part sur les routes, vers l'URSS, pays de cocagne que le communiste de Vent d'Est décrivait aussi comme tel aux paysans.

En septembre 1939 Khoma Gabrys et les autres paysans ukrainiens sont enrôlés dans une guerre mal menée et absurde. La défaite est inévitable et les uhlans de l'armée polonaise se vengent en incendiant le village de Khoma. L'Armée rouge intervient alors comme un deus ex machina capable d'accomplir ce dont étaient incapables les forces sociales endogènes : mettre fin au pouvoir seigneurial, réaliser l'unité du peuple ukrainien avec ses frères soviétiques et donc offrir une perspective nouvelle aux victimes de l'ancien régime. Comme un Vent d'Est, les chars à l'étoile rouge surgissent pour arrêter la folie pyromane des uhlans. La guerre de 1939 apparaît donc comme une catastrophe terminale pour la « Pologne féodale » mais aussi comme un espoir de rédemption pour le peuple. Et «Le Rêve » se réalise quand Anna réapparaît au moment de l'annexion des territoires à l'URSS : elle "revient de là-bas » où elle est devenue une ouvrière accomplie. Dans le décor expressionniste du film, la photo de son usine montre le fonctionnalisme socialiste face au chaos capitaliste. Les deux films se concluent donc sur un happy end forcé par la puissance soviétique.

L'ossature idéologique de ces films est si simpliste qu'elle peut être aisément retournée sans perdre sa cohérence. On le voit dans Zigmund Kolosovski, tourné en 1945. Le gouvernement polonais pro-soviétique a pris les rênes du pays l'année précédente, et, loin de critiquer la nation polonaise, il faut au contraire vanter l'esprit de résistance de ce nouvel allié. Les marqueurs visuels de la polonité n'ont guère changé depuis 1939-1941. On retrouve dans le film le catholicisme (et l'architecture gothique qui va avec [fig. 06]), ainsi que la konfederatka ${ }^{7}$, sauf qu'ils sont désormais connotés positivement. Les prêtres s'opposent aux nazis et le héros revêt un uniforme chamarré pour prendre la radio nationale aux collaborateurs et proclamer l'indépendance nouvelle [fig. 07]. La szlachta et autres composantes des classes dirigeantes ont disparu alors que des paysans « authentiquement » polonais en costume assistent le héros, aux

$6 \quad$ Notons qu'ils sont tous deux ukrainiens.

7 Couvre-chef à quatre pointes, symbole national depuis la Confédération de Bar (1768) et l'insurrection de 1863 . 
côtés de citadins représentant les ouvriers et les intellectuels [fig. 08]. Pourtant, s'il y a ainsi à l'écran un « peuple polonais » œuvrant à sa libération, l'essentiel du travail est fait par le héros. Tout au long du film, il se déguise (en soldat allemand, en officier hongrois... tous ridicules [fig. 09]) et frappe l'ennemi. Complétant l'action du superhéros, l'Armée rouge intervient à la fin et sauve une nouvelle fois la Pologne. La morale de l'Histoire de 1944 est finalement la même que celle de 1939.

Le succès extraordinaire de ce dernier film, qui n'est pas le meilleur du corpus, pose la question de la diffusion et de la réception de ces films. Les spectateurs étaientils sensibles au contenu idéologique de Zigmund Kolosovski au-delà de l'attrait des multiples rebondissements? Le Rêve faisait-il partager sa vision de la dépravation capitaliste ou au contraire instillait-il le désir de lieux de consommation éclairés par des enseignes lumineuses ? Le paysan ukrainien de Vent d'Est suscitait-il une solidarité de classe ou un sentiment de communauté ethnique ${ }^{8}$ ? D'ailleurs, qui a vu ces films parmi les populations concernées ? Si l'on peut douter qu'ils aient été projetés dans l'État polonais reconstitué après la guerre, on ne peut malheureusement pas dire grand-chose de leur diffusion en Union soviétique. Nos sources ne donnent pas d'informations à ce sujet, ni a fortiori sur l'avis des spectateurs.

Nous disposons par contre d'éléments pour tenter de comprendre ce que les créateurs et les participants de ces projets avaient mis d'eux-mêmes dans la production de ces représentations. Nous étudierons ainsi leurs propres expériences interculturelles et transnationales pour voir comment elles avaient préalablement nourri leur travail cinématographique. De ce point de vue, on peut distinguer trois catégories de protagonistes en fonction de leur situation en septembre-octobre 1939: ceux qui étaient déjà citoyens soviétiques, ceux qui le sont devenus car ils vivaient dans les territoires annexés, ceux qui ont choisi de le devenir en se déplaçant vers l'est.

\section{Des films élaborés...}

\section{1 par des Soviétiques...}

Dans ses souvenirs, Mikhail Romm a souligné l'importance de la trajectoire personnelle des artistes qui ont contribué au film Le Rêve :

Ma famille est originaire de Vilno, mon grand-père était propriétaire d'une imprimerie. Et, malgré le fait que je sois né en Sibérie, je suis allé plusieurs fois à Vilno avant la révolution. Evgueni Gabrilovitch [le scénariste] connaît également bien la petitebourgeoisie. [...] Nous avons eu beaucoup de chance avec les acteurs. M. Astangov vivait en Pologne avant la révolution où son père était en poste. Ada Voïtsik est

8 Voir quelques exemples de « mésusages » d'un film par ses spectateurs dans Rittersporn $2014: 194,282$. 
polonaise. Aussi bien Faïna Gueorguievna Ranevskaïa que Rostislav Ianovitch Pliatt comprenaient non seulement la nature sociale mais aussi nationale du film Le Rêve. C'est peut-être pourquoi ils réussirent à trouver les détails vivants, sensibles qui aidèrent à faire de ce film un jalon de mon parcours vers une nouvelle dramaturgie et une nouvelle façon de réaliser des films (Romm 1981 : 328).

On voit que, trente ans plus tard, Mikhaïl Romm présente toujours une situation sociale ethnicisée comme le faisait son film. Évoquant la judéité de biais, il l'associe à la petite-bourgeoisie que son scénariste et lui-même connaîtraient par leur famille. Les racines polonaises de Pliatt, Astangov et Voïtsik et celles, juives, de Ranevskaïa sont censées également leur permettre de comprendre « non seulement la nature sociale mais aussi nationale » de leurs rôles. En citant quatre acteurs du Rêve liés à la Pologne par leur nationalité, leur origine et / ou leur parcours de vie, Mikhaïl Romm suggère que c'était une façon de constituer le casting.

On peut compléter cette liste en étudiant l'équipe des autres films. Zigmund Kolosovski est réalisé par Zigysmund Navrotski, né à Varsovie en 1903, aidé de Boris Dmokhovski [fig. 10], l'acteur principal, dont le nom indique également des racines polonaises. Si la comtesse polonaise et l'institutrice ukrainienne de Vent d'Est sont jouées par des actrices russes (respectivement Olga Jizneva, la femme du réalisateur, et Tatiana Kondrakova), on retrouve Rotislav Pliatt dans le rôle de l'intendant polonais et surtout Amvrosi Butchma et sa femme Valentina Bjeskaïa pour jouer le couple de paysans ukrainiens [fig. 11]. Monument du théâtre et du cinéma ukrainiens depuis les années 1920, Butchma est un authentique Galicien, un natif de Lvov, passé en Ukraine de l'Est à l'occasion de la Première Guerre mondiale et donc devenu citoyen soviétique. Issu d'une famille modeste et ayant exercé mille métiers quand il ne pouvait pas vivre de son art, il avait pu également mûrir la «nature sociale » de ses rôles d'exploités (Velymčanycja 2009).

Néanmoins, si l'on creuse au-delà des propos de Mikhaïl Romm sur son équipe, on peut sérieusement remettre en cause l'adéquation entre l'expérience culturelle des participants et la « nature nationale » des projets de films. Les apparences peuvent être trompeuses. Peut-être juifs, Ranevskaïa, Romm et Gabrilovitch avaient grandi en Russie et avaient baigné dans la culture russe, loin de la « zone de résidence »9. Quant aux « Polonais »... Navrotski était certes né à Varsovie, mais Domkhovski était natif de Saint-Pétersbourg et Pliatt de Rostov-sur-le-Don. De plus, ces trois hommes ainsi qu'Ada Voïtsik avaient tous grandi et fait carrière en URSS et il est même douteux qu'ils aient parlé polonais (Peterburgskij Nekropol' 2013 ; Kuz'mina 1967). Le seul dont on est sûr qu'il maîtrisait la langue de Mickiewicz est le très russe Astangov (Astangov $1971: 247$ ). 
Rostislav Ianovitch Pliatt raconte dans ses mémoires que son père était un Polonais « très russifié » et qu' « on n'entendait pas de polonais à la maison ». L'acteur avait lui-même polonisé son patronyme d'Ivanovitch en Ianovitch et rajouté un « $\mathrm{t}$ » à son nom de famille au début de sa carrière en espérant que cela se remarquerait mieux sur les affiches de théâtre (Pliatt 2000 : 17, 28). On peut penser que la présence au générique des Voïtsik ou Dmokhovski répondait à la même logique et que, pour défendre un projet de film "polonais » devant une bureaucratie élevée à l'époque de la korenizacija ${ }^{10}$, il était de bonne politique de présenter des patronymes sonnant bien. Cette couleur locale n'allait même pas jusqu'à garder les accents : dans tous ces films, les « Polonais » parlent un russe parfait et seul Butchma, le paysan ukrainien dans Vent d'Est, peut s'autoriser une prononciation et des expressions idiomatiques.

On ne peut néanmoins réduire l'influence de la Pologne sur les artisans de ces films à d'éventuelles racines. La découverte par les Soviétiques des territoires arrachés à la Pologne en septembre 1939 est une expérience récente et marquante. Là encore, Mikhaïl Romm l'explique :

À l'automne 1939, j'ai été envoyé en Biélorussie occidentale comme membre de l'équipe cinématographique du front. Au moment de la révolution d'Octobre, je n'avais que 17 ans. Je ne connaissais l'autre monde que par les journaux et les livres. Je ne savais tout simplement pas ce qu'était un magasin ou hôtel privés. Et voici que je me retrouvais au cœur d'une province petite-bourgeoise, qui m'étonnait par des relations entre les gens, une relation à l'argent et des buts dans la vie si différents. De plus, j'étais tombé au fin fond de la campagne polonaise au moment du désastre. Tout ce qui se passait était proprement extraordinaire. Les impressions des premiers jours furent particulièrement fortes. [...] J'y ai passé près de deux mois et la force de ce que j'ai éprouvé fut énorme. C'était un voyage non seulement dans l'espace mais aussi dans le temps (Romm $1971: 323,326)$.

On voit ici la conjonction des trois facteurs qui forment l'image de la Pologne pour Mikhail Romm : une culture et une histoire polonaises (qu'il n'évoque pas dans cet extrait), une économie et une société marchandes (ersatz de capitalisme pour quelqu'un qui a grandi en URSS), une situation de chaos provoquée par la guerre. Le réalisateur rencontre à ce moment-là Evgueni Gabrilovitch, correspondant des Izvestia, qui deviendra son scénariste pour Le Rêve, et les deux hommes partagent les mêmes impressions. Là où Mikhaill Romm rencontre un pédiatre réduit à vendre des pommes, Gabrilovitch a acheté de la limonade à un ingénieur obligé de travailler dans le magasin de sa mère, ainsi décrite : « pas bête, grosse, de l'asthme » (Romm 1971 : 323 ; Kinojudaica 2009 : 85). L'idée de départ du Rêve est là toute entière.

10 Littéralement « enracinement ». Dans les années 1920, politique soviétique de recrutement et de promotion de cadres locaux dans les républiques et régions autonomes définies comme des entités nationales selon des critères culturels et ethniques. 
Dans la série d'entretiens qu'il donne peu avant sa mort en 1993, Gabrilovitch nuance un peu la vision très négative de la Pologne que donnait Mikhaïl Romm vingt ans plus tôt. À la fois, il continue d'affirmer qu'il voulait que les Soviétiques « comprennent ce qu'était l'Europe de ces années-là », marquée par la crise et la guerre. En même temps, ce premier " pas en Europe » lui fait découvrir également la diversité des types humains qui nourriront son écriture : " commis voyageurs, prostituées, millionnaires, pauvres... ». Il est aussi confronté à une profusion de marchandises qu'il ne soupçonnait pas. Il se souvient ainsi d'avoir fait l'acquisition de son premier imperméable dans le véritable « caravansérail » qu'était pour lui la Pologne de 1939 (Korjakov 1998 - 4 : 10'44”-19'20”). Les impressions produites par la rencontre avec la Pologne « féodale et bourgeoise » sont ambivalentes et font donc l'objet d'une sélection avant d'être transposées à l'écran. De la société marchande, on retiendra les relations humaines qu'elle induit et non l'aisance relative qu'elle permet.

\section{2 ... par des citoyens polonais soviétisés malgré eux}

Et les citoyens polonais précipités dans l’Union soviétique en septembre 1939 ? Officiellement, ils ont été consultés démocratiquement et leurs représentants élus ont voté la demande de rattachement à l'URSS lors de « l'Assemblée populaire » tenue à Lvov en octobre 1939. En fait, les autochtones ont joué les utilités dans une pièce écrite par Moscou (Gross 2002 : 91 et ss.). Comme ces nouveaux « Ukrainiens de l'ouest » ont fait de la figuration politiquement, on s'attendrait à en retrouver dans des petits rôles dans les films de 1940-1941. Cela ne se vérifie que dans Vent d'Est. À la fin du générique, après le nom des vedettes (Butchma, Jizneva, Pliatt...), on lit :

Acteurs des théâtres polonais et ukrainiens des régions occidentales d'Ukraine :

\begin{tabular}{|c|}
\hline .............E. Kurilo \\
\hline ...S. Stadnikivna \\
\hline ...O. Slipenki \\
\hline Ivan (frère de Khoma).... \\
\hline ...L. Serdiukova \\
\hline Doubtchak....... \\
\hline ..V. Ratchka \\
\hline
\end{tabular}

Même si nous n'avons rien pu trouver sur L. Serdiukova, ce que les rares sources disponibles nous permettent d'apprendre sur le parcours des autres acteurs du cru n'est pas sans intérêt [fig. 12 \& 13].

On constate déjà que parmi les « acteurs des théâtres polonais et ukrainiens des régions occidentales d'Ukraine », la balance n'est guère équilibrée avec cinq Ukrainiens avérés pour un seul Polonais. Il s'agit de Władysław Ratschka (ou Ratschka-Sowiński) qui avait commencé sa carrière théâtrale à Lwów en 1895 pour 
jouer ensuite sur toutes les grandes scènes de Pologne (Varsovie, Cracovie...) pendant quatre décennies. L'essentiel de son activité après 1918 était centré en Volynie (Łuck) et Galicie (Lwów), c'est-à-dire exactement les futures « régions occidentales d'Ukraine » (Maleončuk 2015 : 126 ; Encyklopedia teatru polskiego sd). Sa présence dans le film Vent d'Est pour jouer un Polonais, Iouzef / Józef, n'a donc rien d'anormal. Il est cependant piquant de découvrir qu'il s'agissait de sa seconde apparition au cinéma. La première fois, il incarnait un petit rôle dans Les Dix de Pawiak (Ryszard Ordyński, Dziesięciu z Pawiaka, 1931), film patriotique qui exaltait l'action des terroristes polonais contre les dignitaires... russes en 1905 (Film polski sd). On peut penser qu'entièrement dévoué à son art sans avoir jamais été une vedette, il acceptait toutes les propositions, sans prêter attention à l'idéologie des productions ${ }^{11}$. L'attitude sans doute assez opportuniste du comédien polonais sous les soviets peut aisément s'expliquer par le risque social qu'il y avait à ne pas être de la nouvelle nationalité titulaire.

Les acteurs ukrainiens auraient dû avoir des raisons plus profondes d'adhérer au projet culturel soviétique. Après tout, la pression des autorités polonaises contre l'expression ukrainienne qu'illustrait Vent d'Est était réelle (Kappeler 1997 : 160161). Néanmoins, 1939 ne semble pas accélérer la carrière de comédiens qui avaient déjà un statut d'artiste reconnu. Iossip (Ossip) Stadnyk fut acteur puis directeur et régisseur du théâtre ukrainien de Lwów « Ruska Besida » dès avant 1914. Il y avait fait travailler Petro Soroka et c'est lui qui avait mis le pied à l'étrier au jeune Butchma en 1905. On peut penser que ce dernier, devenu soviétique dès 1919, fit le trait d'union avec son ancien maître de Lwów en 1940 qui lui-même constitua le reste de la troupe « locale » à partir de son réseau de connaissances. En effet, Stefa Stadnykivna est sa fille. Elle fit ses études à l'École d'art dramatique de Varsovie avant de rejoindre le théâtre de son père et de se produire également sur les scènes de Poznan, de Wilno ou de Kharkov. De la même génération que cette dernière, Evguen Kurylo et Orest Slipenki avaient quant à eux exercé leur talent dans les troupes ukrainiennes de Stanisławów (Stanislav, aujourd'hui Ivano-Frankivsk). Toutes les institutions théâtrales d'Ukraine de l'ouest avaient été nationalisées par les Soviétiques dès l'automne 1939. Le théâtre d'Ossip Stadnyk à Lviv avait pris le nom de Lessia Ukraïnka et avait pris ses quartiers à l'opéra alors qu'à Stanislav il était baptisé Ivan Franko ${ }^{12}$ (TES ; Maksymenko 2006 : 14 ; Mandzjuk sd).

11 Il est mort à Lviv en 1944, quelques mois après la reconquête de la ville par les Soviétiques. On ne sait rien de son attitude pendant l'occupation nazie et de ce qui lui est arrivé à la « Libération ».

12 Il s'agit de deux écrivains ukrainiens du tournant du siècle, des " démocrates révolutionnaires » selon la terminologie soviétique. 
La soviétisation par en haut, cette "révolution de l'extérieur 》 (Gross 2002), semblait couronnée de succès. Avec la prise en main des théâtres, les Soviétiques avaient constitué un vivier d'acteurs dans lequel puiser pour contribuer à l'effort propagandiste accompagnant l'annexion. Les nazis feront de même dans la période suivante. En effet, tous les acteurs ukrainiens sont restés sur place et souvent restés en place après l'attaque allemande du 22 juin 1941. Kurylo, Soroka, Slipenki et Stadnykivna travaillent à l'opéra de Lvov (qui avait donc repris son nom d'avantguerre mais était resté à dominante ukrainienne, alors que la ville faisait désormais partie du Gouvernement général de Pologne, sous administration allemande). Seul Stadnyk connaît quelques difficultés : en 1942, il perd son poste à l'opéra et ne retrouve un travail stable que l'année suivante, comme directeur d'un théâtre à Drohobytch (Maksymenko 2001, 2005, 2006 \& 2013).

Professionnellement, le changement de maîtres a été une simple péripétie pour la plupart des acteurs : Soroka, Slipenki, Stadnykivna et Stadnyk ne bougent pas plus en 1944 qu'ils n'avaient bougé en 1941. De nouveau soviétiques, les comédiens travaillent dans de grandes institutions de Lviv : Stadnyk au théâtre Lessia Ukrä̈nka comme en 1939, sa fille au Théâtre du jeune spectateur et Soroka au théâtre Maria Zankovetska. Stadnyk et Soroka sont finalement rattrapés par leur passé en 1947 : des agents du MVD les arrêtent. Soroka est accusé de « s'être volontairement mis au service des occupants allemands en qualité de sous-directeur du théâtre dramatique et [d'avoir] pris part à la représentation de la pièce anti-soviétique «Le Triomphe du procureur Dalski» aussi bien à Lvov que dans les villes voisines » (Maksymenko $2006: 16)$.

Concernant Stadnyk, qui avait objectivement moins collaboré " grâce " à son licenciement de l'opéra, il lui est tout de même reproché son enthousiasme proallemand en juin 1941 alors qu'il avait été élu député au Soviet suprême de la république d'Ukraine en mars 1940... Ils sont tous les deux déportés. Seul Stadnyk reviendra à Lviv pour y mourir en 1954, quelques mois après sa libération (Maksymenko 2001, 2006). Sans qu'on sache pourquoi, Stadnykivna et Slipenki échappent à la répression. La première poursuit une carrière théâtrale honorable à Kharkiv et Lviv jusqu'à la fin des années 1970 (TES). Difficile de savoir ce qu'est devenu le second, si ce n'est qu'ironiquement il apparaît en 1981 au générique d'un film soviétique sur la lutte fratricide entre communistes et nationalistes dans les Carpates ukrainiennes (Vladimir Denisenko, Vysokij Pereval, cf. Anotovanyj... 2011 : 55).

C'est un sujet qui aurait sûrement intéressé Evhen Kurylo. Ayant suivi la retraite nazie comme la majorité de ses collègues de l'opéra de Lvov, il est membre de l'Ensemble des acteurs ukrainiens réfugiés en Allemagne de l'Ouest (Revuc'kyj 2006 : 200). Émigré ultérieurement aux États-Unis, il est actif dans la diaspora nationaliste. Dans la revue de l'OUN (Organisation des nationalistes ukrainien, bandériste), il évoque l'attaque allemande de 1941 comme « un criminel [...] venu s'en prendre à un 
autre criminel ». Sur la période 1939-1941, il ironise : c'est une « «libération» par la horde bolcho-moscoutaire » (Kurylo 1977 : 931). Dans Vent d'Est, ce même Kurylo jouait Andreï, le jeune bolchevik intrépide qui élevait la conscience révolutionnaire des paysans et ridiculisait les pandores polonais.

Si le projet soviétique d'intégrer idéologiquement le milieu culturel autochtone a visiblement échoué, on ne peut pourtant pas conclure à un rejet massif de la part des comédiens ukrainiens de la vie sous le régime des soviets. La conquête nazie avait été brusque et inattendue, laissant chacun adopter une tactique de survie à court terme. Par contre, quand la défaite des troupes allemandes se profilait, il était loisible de prendre la tangente comme Kurylo. Il est néanmoins le seul de l'équipe de Vent d'Est à voir son salut personnel et professionnel dans une fuite à l'Ouest.

\section{3 ... par une Soviétique par choix, Wanda Wasilewska}

Parmi les ex-citoyens polonais devenus soviétiques, un parcours fait exception, celui de Wanda Wasilewska. Elle apparaît au générique de Vent d'Est comme «Conseil pour le scénario ». C'est une écrivaine polonaise qui publiait depuis 1934 mais qui n'était pas une personnalité de premier plan avant 1939. Son ascension et son assimilation après l'annexion sont spectaculaires. Pour ne prendre que le domaine cinématographique, Vent d'Est n'était que sa deuxième expérience. En 1936, elle avait été co-scénariste d'un documentaire d'Aleksander Ford consacré à un sanatorium pour enfants du Bund, le parti socialiste juif (Droga mlodych / Nous arrivons ${ }^{13}$ ). En 1940, elle conseillait les scénaristes soviétiques pour évoquer les régions qui venaient d'être retranchées à la Pologne. Elle est de nouveau scénariste du film L'Arc-en-ciel de Mark Donskoï qui évoque la résistance d'un village ukrainien à l'occupation nazie. C'est un succès mondial en 1944 alors que l'Allemagne hitlérienne chancèle ${ }^{14}$. Non seulement elle rencontre alors la notoriété, mais elle la doit à un sujet soviétique qui n'a plus rien à voir avec la Pologne. Ce tournant opéré au cinéma reflète l'évolution de sa carrière littéraire. Le scénario du film L'Arc-en-ciel est adapté de son propre roman, publié en 1942 et récipiendaire du prix Staline. Après une série de nouvelles patriotiques de la même veine, elle publie des livres sur la réintégration des anciens combattants soviétiques, qui peinent à retrouver une place tant dans leur couple que dans la société (Par Amour / Prosto ljubov', 1944 ; Ténèbres vaincues / Kogda zagoritsja svet, 1946). Cette dernière œuvre fait la synthèse du roman de guerre et du roman de production et montre à quel point l'auteure a fait sienne une culture spécifiquement soviétique.

13 Le film est diffusé en France sous ce titre pendant le Front populaire (L'Intransigeant, 13.11.1936, p. 9). 
Cette réussite littéraire doit beaucoup à un choix politique et à ses conséquences heureuses pour l'auteur. Militante socialiste en Pologne, elle est partie à pied vers l'est dès le 7 septembre 1939, avec son mari (qui est assassiné quelques temps après). Un mois plus tard, elle est en zone soviétique et gagne rapidement Lvov. Vite publiée dans la presse soviétique, adhérente au PC, elle est élue députée en mars 1940. En avril, à l'occasion de la session du Soviet suprême à Moscou, elle est mandée par Staline qui souhaite la rencontrer (Tokarev 2007 : 42-47). Devenue la meilleure des Soviétiques de la minorité polonaise d'Ukraine en 1940, elle sert dans l'Armée rouge comme cadre politique dès la reprise de la guerre. «Au début 1943, Vassilevskaïa est rappelée du front à Moscou. Là l'attendait une nouvelle tâche d'importance » (Usievič 1954 : 84). Staline veut créer en URSS des institutions polonaises qui puissent faire pièce au gouvernement de Sikorski en exil à Londres. Wanda redevient donc polonaise, mais la meilleure des Polonaises pro-soviétiques : elle participe à la création de l'Union des patriotes polonais $(Z P P)$ puis à la mise sur le pied de guerre de la division Kościuszko qui combat avec l'Armée rouge [fig. 14]. En 1944, quand la Pologne est peu à peu libérée, elle intègre le Comité polonais de libération nationale ( $P K W N)$, gouvernement provisoire soutenu par Moscou. En 1945 pourtant, elle retourne vivre en Ukraine, à Kiev, avec le dramaturge en vue Aleksandr Korneïtchouk, son nouveau mari (Vengerov $1955: 74-75,85$ ). Elle s'y éteindra en 1964. On voit à quel point son passage du côté soviétique en 1939 a fait basculer sa vie : elle a changé de nationalité, de parti et de mari.

Soixante-dix ans plus tard, Marek Edelman, qui l'avait connue avant guerre, restait étonné par ce parcours :

C'était une femme merveilleuse qui faisait de très bons films. Elle était mal vue du gouvernement polonais, c'est pourquoi nous l'aimions beaucoup. C'était une personne merveilleuse. C'est bizarre qu'elle soit devenue communiste quand elle était à l'est ; mais c'est ce qui est arrivé, alors qu'elle était une socialiste normale et une femme formidable. Son mari aussi, qui était au PPS et a été tué par les Russes. Ensuite, elle s'est mise avec ce Korneïtchouk, elle était amoureuse du ministre de la culture ; de la culture, c'est bien ça ? (Grupinska 2008).

Vassili Tokarev a éclairé une partie de ses motivations grâce à la correspondance conservée aux Archives d'État de la littérature et de l'art à Moscou. Douée d'un solide sens institutionnel, elle contacte son éditeur moscovite dès son arrivée en territoire soviétique puis accède au milieu des écrivains envoyés en Ukraine de l'Ouest comme correspondants de guerre. Elle ne refuse pas les contraintes politiques (adhésion au $\mathrm{PC}$ et députation) qui accompagnent la reconnaissance professionnelle. À un niveau plus personnel, elle sait oublier l'assassinat de son mari par des « inconnus ${ }^{15}$ »; elle sait se lier à un homologue, un écrivain ukrainien soviétique en pleine ascension, 
Korneïtchouk ; elle sait aussi se rappeler au bon souvenir de Staline après leur première rencontre (Tokarev 2007).

À Kiev aussi, Wanda Wasilewska a laissé des documents que l'on peut consulter aux Archives centrales - musée de la littérature et de l'art. Quelques dossiers permettent d'affiner le portrait de 《l'écrivaine soviétique polonaise et activiste sociale $»^{16}$ pour comprendre l'articulation de son travail créateur avec sa trajectoire politique et institutionnelle. Il y a d'abord un gros album tenu de 1934 à 1938 où Wanda mesurait l'avancée de sa carrière en collant des coupures de presse la concernant. Elle devait y être attachée car, s'il s'est conservé, c'est qu'elle l'a emporté avec elle sur les routes de l'exil à l'automne 1939 ou l'a fait récupérer par la suite.

Son premier roman, qui évoque les classes paupérisées des villes, Oblizce dnia (Le Visage du jour), est le plus souvent recensé au milieu d'autres ouvrages de gauche qui ne plaisent guère aux critiques de la grande presse. Ils attaquent cette « littérature prolétarienne » qu'ils soupçonnent de sympathie pour Moscou. Autre angle d'attaque, le genre : «Des femmes écrivent », «L'attaque des femmes ». Tout cela est brocardé comme un "radicalisme de salon ». Quant à Wasilewska spécifiquement, on lui reproche la «partialité » et la « forme naïve » de son livre. «Roman ou reportage ? » demande un autre critique, peu convaincu par le souci de réalisme de l'auteur. Seuls les journaux socialistes polonais (Glos poranny, Myśl socjalistyczna) louent ses livres. Les Soviétiques aussi apprécient cette littérature engagée et réaliste. Le livre est chroniqué par la Literaturnaja Gazeta en avril 1935 et, au mois d'août, elle reçoit de Moscou une lettre de la revue La Littérature internationale, organe de l'Union internationale des écrivains révolutionnaires, lui proposant de la publier. Elle est bientôt traduite en russe. C'est le début d'une renommée internationale dans les milieux de gauche, laquelle se confirmera avec le film Droga młodych, projeté dans plusieurs pays par des réseaux militants. La réussite ne la laisse visiblement pas indifférente : d'une photo de presse à l'autre, Wasilewska semble de plus en plus apprêtée et les deux derniers portraits tirés en 1938 sont assez glamour [fig. 15 ${ }^{17}$.

La femme de 35 ans qui arrive à Lviv en 1939 a des atouts pour commencer une carrière en URSS. Elle connaît bien les milieux militants où elle est reconnue non seulement pour ses publications mais aussi pour son engagement (plusieurs grèves durement réprimées l'ont vue comme participante ou soutien actif). Sans être au PC, elle s'en était rapprochée politiquement à l'occasion de la politique de Front populaire, mais n'avait pas partagé la disgrâce et l'élimination des communistes polonais par Staline (Shore $2006: 109$ ). Le fonctionnement de l'establishment soviétique avec

16 Comme l'indique la couverture de l'un d'entre eux.

17 CDAMLM (Kiev), F. 73, op. 1, d. 761 : Wanda Wasilewska, « Recenzii », 1934-1938, 11. $1-75,158$ et ss. 
lequel elle était déjà en contact pour des questions éditoriales ne lui est donc pas étranger : il est normal pour elle que la valeur d'un écrit soit indissociablement littéraire et politique et que sa diffusion relève de choix d'appareil. Cela se confirme à la lecture d'un dossier qui rassemble ses articles de 1939-1940. Dans ses louanges de la politique soviétique, elle sait passer progressivement de l'exaltation de l'émancipation à celle de la puissance. Nous seulement elle célèbre de plus en plus l'Armée rouge d'une année sur l'autre, mais sur les thèmes sociaux elle infléchit aussi son discours. Jusqu'au début 1940, elle évoque plusieurs fois l'antisémitisme qu'avaient subi les Juifs en Pologne alors qu'ultérieurement les affres de l'ancien régime n'apparaissent plus que sous la forme d'une lutte des classes simpliste entre des ouvriers vertueux et des patrons dépravés ${ }^{18}$. Sa capacité d'adaptation est également personnelle. On l'avait vue soigner sa mise en tant qu'auteure de plus en plus connue sur le marché éditorial en Pologne. En URSS, elle arbore au contraire une apparence austère : l'artiste est au service du peuple [fig. 16].

La rectitude politique et morale était une condition nécessaire mais pas suffisante pour devenir consultante pour le scénario de Vent d'Est. Wanda Wasilewska avait d'autres titres à faire valoir. Après le livre sur le prolétariat urbain en 1934, elle avait continué de publier dans la même veine sociale et contestataire, cette fois sur les campagnes : ouvriers agricoles (Ojczyzna / La Patrie, 1935) et petits paysans (Ziemia w jarzmie / La Terre sous le joug, 1938) aux prises avec des grands propriétaires souvent nobles, prompts à instrumentaliser le nationalisme. Capable de jouer sur les ressorts du drame paysan, l'intellectuelle cracovienne avait également des prédispositions sur la question des confins orientaux de la Pologne. Son père, militant socialiste de l'ancienne génération et proche de Piłsudski, était spécialiste des kresy et particulièrement de la Galicie et de l'Ukraine. Il espérait une intégration des minorités à la nation polonaise grâce à l'autonomie, culturelle, que leur aurait prodiguée la Rzeczpospolita restaurée (Symonenko 2003). Sa fille connaît sûrement ses articles sur la question et elle était intervenue en 1936 au Congrès des travailleurs de la culture qui s'était tenu en Galicie en apportant son salut «à Lwów l'Ukrainienne ». Elle avait alors côtoyé des communistes qui défendaient déjà le rattachement de ces régions ukrainiennes et biélorusses aux républiques soviétiques éponymes (Shore 2006 : 128).

Qu'en pensait-elle au début 1939, alors qu'elle travaillait à un roman sur la politique de l'État polonais dans ces confins (Plomień na bagnach / La Flamme sur les marais, 1940) ? En tout cas, la guerre infléchit définitivement son opinion à l'automne. On le lit dans ses articles. Elle attribue la déroute de l'armée polonaise à la trahison des 
pany, du gouvernement et des officiers. Elle retrace aussi sa fuite vers l'est. Exténué, le groupe de réfugiés dont elle fait partie s'est arrêté aux rives de la Stochod.

Et voilà que la frontière se déplace vers nous, nous embrasse ; cette frontière vers laquelle nous nous précipitions pendant ces nuits terribles aux lueurs d'incendie ; cette frontière vers laquelle nous marchions, les pieds blessés et sanglants, les yeux épuisés par l'insomnie [...] Mais la frontière se déplace, elle apporte à la terre ukrainienne la paix et aux désespérés le salut ${ }^{19}$.

Elle incrimine la haine entre nations qui régnait en Pologne, l'antisémitisme on l'a dit -, mais aussi les restrictions contre les langues et cultures ukrainiennes et biélorusses, qu'elle développe en montrant comment l'école polonaise déformait l'histoire, ukrainienne notamment. Tous les thèmes du film d'Abram Room sont déjà présents, jusqu'au titre « Vent d'Est » qu'elle utilise pour célébrer le rattachement de la Boukovine et de la Bessarabie en juin $1940^{20}$. On ne sait pas précisément comment Wanda Wasilewska fut associée au projet de film ni quel y fut son rôle précis, mais, au-delà de la communion idéologique, les points de contact potentiels avec l'équipe cinématographique étaient désormais nombreux. Korneïtchouk qui a été chargé de rassembler les intellectuels polonais progressistes à Lviv peut faire l'interface avec les milieux culturels soviétiques. Wanda est devenue conseiller littéraire du théâtre polonais de Lviv après l'arrestation de son prédécesseur, le poète Broniewski (Shore $2006: 154,158-159,172)$.

Le même cocktail de sincérité, d'opportunisme et de jeu des circonstances se retrouve deux ans plus tard lors de l'élaboration du film Raduga / L'Arc-en-ciel. Signe de son statut, Wanda a été évacuée de Lviv en juin 1941 par un véhicule envoyé exprès $^{21}$. Ce n'est pas un prétexte à lâcheté puisqu'elle entre rapidement dans l'Armée rouge en qualité d'instructeur politique. À ce titre, elle participe à la rédaction du journal Za radians'ku Ukraïnu (Pour l'Ukraine soviétique) destiné aux partisans (Vengerov 1955 : 64) [fig. 17]. La Polonaise, devenue une Ukrainienne soviétique, doit donc écrire sur la résistance dans sa nouvelle patrie occupée. Son nouveau mari, Korneïtchouk, travaille avec elle et sur les mêmes sujets. Au début 1942 on monte sa pièce Les Partisans dans les steppes d'Ukraine (Partizany v stepjax Ukrainy) qui montre l'occupation à l'échelle d'un village, avec des caractères tranchés de partisans, de collaborateurs et d'Allemands. L'œuvre est rapidement adaptée au cinéma par Igor

$19 \quad$ Ibid., 1.16.

$20 \quad$ Ibid., 11. 23-27, 118.

21 Au grand dam d'Ossip Stadnyk qui donne cette inégalité de traitement comme explication quand les agents du MVD lui demandent pourquoi il n'a pas quitté la ville lors de l'attaque nazie (Maksymenko $2001: 22$ ). 
Savtchenko et sort sur les écrans en mars 1943, avec Natalia Ujviï dans le rôle féminin principal (Anotovanyj $2011: 273$ ).

En février 1942, la Pravda avait publié un court reportage d'Oskar Kourganov (Esterkine) sur une villageoise de la région de Moscou qui avait préféré voir mourir son nouveau-né et mourir elle-même plutôt que de dire aux Allemands où les partisans étaient cachés (Kurganov 1942). Wanda Wassilewska s'adresse au journaliste pour demander l'autorisation de s'inspirer du récit ${ }^{22}$. En juin 1942, elle obtient un congé pour écrire son roman qui est publié en feuilleton dès l'automne dans les Izvestija (Usievič 1954 : 76-77). L'histoire de la résistance d'un village d'Ukraine est centrée sur le personnage d'une femme enceinte, inflexible malgré les tortures, qui meurt avec son enfant plutôt que de parler. Le livre, parfaite synthèse de la pièce de son mari et du reportage de Kourganov, reçoit le prix Staline en 1943. Wanda Wasilewska a terminé son adaptation en scénario en janvier ${ }^{23}$, juste avant de redevenir une « patriote polonaise » pour Staline. Le film est tourné dans la foulée par Mark Donskoï à Achkhabad, avec Natalia Ujviï encore dans le rôle principal [fig. 18].

$$
* * *
$$

La capacité de Wanda Wasilewska à être alternativement polonaise ou soviétique (Mrozik 2013) et à intervenir avec autant d'efficacité dans les deux cultures montre $a$ contrario l'échec massif des transferts culturels entre Pologne et URSS. La politique menée par les Soviétiques semble en effet aboutir à une impasse quand on l'étudie par le prisme du cinéma. Elle visait à intégrer les régions annexées au grand récit soviétique et à amalgamer anciens et nouveaux citoyens de l'URSS dans le milieu du cinéma. Contrairement à ce que le cinéma soviétique avait réussi à faire pour la guerre civile, les films étudiés ici n'ont pas créé d'épopée capable de s'imposer comme images-mémoire de l'événement. Vent d'Est et Zigmund Kolosovski sont des films de genre entravés par l'idéologie alors que Le Rêve, qui est d'ailleurs la seule tentative d'aborder la question juive, s'éloigne des contingences locales pour atteindre à l'universel en décrivant le jeu des passions humaines. En somme, les films sont d'autant meilleurs qu'ils évacuent le sujet polonais proprement dit.

Les Soviétiques avaient conçu des œuvres en couleur locale qui recyclaient des clichés plus qu'elles ne créaient un type original, et les origines juives ou polonaises des créateurs n'y changeaient pas grand-chose. Quant aux ex-Polonais associés à un projet soviétique, leur soviétisation avait été de façade à l'image de celle de l'ensemble

22 Nacional'na kinostudija hudožnih fil'miv imeni Oleksandra Dovženka - Muzej (Musée du studio national cinématographique d'art Oleksandr Dovjenko, Kyïv), Dossier «Raduga» (sur la réception du film) : V. Bulyčëva, [sur le sacrifice d'Aleksandra Martynovna Drejman - texte dactylographié, sd.], 1. 7. dossier « Wanda Wasilewska, Raduga - Literaturnyj scenarij », 1943. 
de ces régions. Ils exerçaient leur métier en Pologne avant-guerre, ils avaient continué à le faire sous les soviets et ils poursuivraient leur activité à l'heure allemande. Il ne faut d'ailleurs pas exagérer leur engagement. Seul Evguen Kurylo a ouvertement sympathisé avec les nationalistes, mais dans l'émigration après 1945. Rien ne prouve qu'il fut militant dans l'âme auparavant. En quoi Wanda Wasilewska se différenciaitelle de ses concitoyens ? Certes, elle était sûrement idéologiquement plus proche des Soviétiques que les acteurs ukrainiens.

On pourrait cependant trouver des convergences entre les comédiens ukrainiens et l'écrivaine avant 1939. Ils font tous partie des milieux culturels de la Pologne d'avantguerre, mais il y occupent une situation marginale, Wasilewska de par ses positions politiques, les Ukrainiens à cause de leur appartenance à une minorité opprimée. Néanmoins, il y a une grande différence entre leurs modes d'activité professionnelle. Les comédiens travaillent dans des structures privées et, tant que le public paie pour les voir, ils gagnent leur vie, quel que soit le caractère idéologique du spectacle. Pour Wasilewska, le réalisme socialiste est au contraire une nécessité civique et esthétique bien avant la soviétisation. Son œuvre est donc trop clivante pour une diffusion purement marchande et sa notoriété se développe dans les réseaux militants en Pologne d'abord puis à l'échelle européenne. En 1939, les Soviétiques changent les règles du jeu culturel en nationalisant les institutions et en privilégiant le contrôle politique et non les recettes pour créer une nouvelle élite culturelle. Les comédiens ukrainiens sont pris à contrepied (d'où leur soulagement sous la domination nazie qui restaure le théâtre privé - Maksymenko 2013 : 68) alors que Wanda Wasilewska se retrouve dans son élément.

Quand Mikhaïl Romm affirmait qu'avant de découvrir les régions de Pologne occupées par l'Armée rouge il " ne savai[t] tout simplement pas ce qu'était un magasin ou hôtel privés », il soulignait la différence fondamentale de « culture » entre ce pays et le sien. Elle ne résidait pas dans des références artistiques ou littéraires spécifiques mais dans un rapport au monde, dans un modèle de civilisation. L'ayant adopté, Wanda Wasilewska pouvait être indifféremment polonaise ou ukrainienne : elle restait « socialiste ", soviétique. Ce type de parcours n'était envisageable que pour qui intégrait les couches dirigeantes soviétiques. C'est pourquoi il n'apparait pas dans les films qui entendaient défendre et illustrer les nouvelles relations soviétopolonaises. Faute de pouvoir valoriser un égalitarisme qui n'est plus de mise en URSS, ces films mettent en exergue les caractères ethniques, culturels au sens étroit du terme, conformément à une idéologie soviétique qui retrouve au même moment des accents nationalistes. Ils s'interdisent par là même de faire dialoguer les cultures. 


\section{Renvois bibliographiques}

Anotovanyj katalog fil'miv Nacional'noï kinostudï hudožnih fil'miv imeni Oleksandra Dovženka 1928-2011, Kiev : Nacional'na kinostudija hudožnih fil'miv imeni Oleksandra Dovženka, 2011.

[Astangov Mihail,] Stat'i i vospominanija o M. F. Astangove. Sost. N. E. Al'tman i O. N. Rossihina, Moscou : Iskusstvo, 1971.

Aunoble Éric, «Faire de l'étranger un Soviétique grâce au cinéma : La soviétisation de l'Ukraine occidentale aux actualités filmées (1939-1949) » in Marie-Christine Autant-Mathieu (dir.), L'Étranger dans la littérature et les arts, Lille : Les presses du Septentrion, 2014, pp. 135-146.

Deak Tamas, "Wind from the East ", Poemas del rio Wang, 12.2011, document en ligne, consulté le 29.06.2016 <http://riowang.blogspot.fr/2011/12/wind-from-east.html>.

Dovženko Aleksandr, Osvoboždenie ukrainskih i belorusskih zemel' ot gneta pol'skih panov $i$ vossoedinenie narodov-brat'ev v edinuju sem'ju [film], Kiev : Kievskaja kinostudija \& Ukrkinohronika, 1940.

Dufour Éric, « Une analyse de Heimkehr (G. Ucicky, 1941)», ILCEA 2015, n² 23, document en ligne, consulté le 4.07.2016 <http://ilcea.revues.org/3393>.

Encyklopedia teatru polskiego, «Władysław Ratschka », document en ligne, consulté le 28.06.2016 <http://www.encyklopediateatru.pl/osoby/40935/wladyslaw-ratschka>.

Film Polski, « Dziesięciu z Pawiaka », Państwowa Wyższa Szkoła Filmowa, Telewizyjna i Teatralna im. Leona Schillera w Łodzi. Internetowa Baza Filmu Polskiego, document en ligne, consulté le 11.07.2016 <http://www.filmpolski.pl/fp/index.php?film=22368>.

Gousseff Catherine, « Médiation et assistance : les anciens réprimés polonais dans l'URSS en guerre », Connexe. Les espaces postcommunistes en question(s), 2015, $\mathrm{n}^{\circ}$ 1, pp. 75-96.

Gross Jan T., Revolution from abroad : the Soviet conquest of Poland's Western Ukraine and Western Belorussia, Princeton N.J. : Princeton University Press, 2002.

Grupinska Anna, Anna Szczesna, Joanna Klara, Agnieszka Zuchowska, « Medem’s Sanatorium - Marek Edelman, Social activist [2003] », London : Web of stories, 2008, document en ligne, consulté le 4.06.2015 <http://www.webofstories.com/play/marek.edelman/11>.

Hicks Jeremy, First Films of the Holocaust : Soviet Cinema and the Genocide of the Jews, 1938-1946, Pittsburgh : University of Pittsburgh Press, 2012.

Himka John-Paul, Socialism in Galicia : The Emergence of Polish Social Democracy and Ukrainian Radicalism (1860-1890), Cambridge Mass. : Harvard University Press, HURI, 1983.

Kappeler Andreas, Petite histoire de l'Ukraine, [1994] Paris : Institut d'Etudes Slaves, 1997.

Kinojudaica, L'Image des Juifs dans le cinéma de Russie et d'Union soviétique des années 1910 aux années 1960, Documents réunis et présentés par Valérie Pozner, Toulouse : Cinémathèque de Toulouse, 2009.

Korš-Sablin Vladimir, Ognennye gody [film], Minsk : Sovetskaja Belarus’, 1939.

Kurganov Oskar, Mat', Moscou : Pravda, Coll. Iz frontovoj žizni, 1942.

Kurylo Evgen, « Jak proliz «verbljud vuhom golki» (Spogad aktora z-pered 40-ka rokiv) », Vyzvol'nyj Šljah (OUN), 1977, n 7-8 (352-353), pp. 928-931.

Kuz'mina Elena, "Ada Vojcik », Aktery sovetskogo kino. Vypusk 3, Red. Tuljakova V., Liščinskij I., Moscou : Iskusstvo, 1967. 
Magocsi Paul Robert, The Shaping of a National Identity : Subcarpathian Rus', 1848-1948, Cambridge, Mass. : Harvard University Press, 1978.

Maksymenko Svitlana, «Sprava jogo žyttja », Teatroznavčyj žurnal « Proscenium », 2001, $\mathrm{n}^{\circ} 1$, pp. 20-35.

Maksymenko Svitlana, «Try vystavy Ivana Ivanyc'kogo », Teatroznavčyj žurnal « Proscenium », $2005, n^{\circ} 1-2$, pp. 17-24.

Maksymenko Svitlana, « «Ključi» Petra Soroky (Epizody žyttja ta tvorčosti mytcja) », Teatroznavčyj žurnal « Proscenium », 2006, n 15-16 (2-3), pp. 14-21.

Maksymenko Svitlana, «Teatral'ne žyttja L'vova 1941-1944 rr. », Naukovyj visnyk Kyïvs 'koho nacional'noho universytetu teatru, kino i telebačennja imeni I. K. Karpenka-Karoho, 2013, n 13 , pp. 67-84.

Maleončuk Halyna, Suspil'no-polityčnyj, social'no-ekonomičnyj ta kul'turnyj rozvytok luc'ka u mižvojennyj period (1919-1939rr.), Luc'k : Dysertacija na zdobuttja naukovoho stupenja kandydata istoryčnyh nauk (Naukovyj kerivnyk : Anatolij Švab), 2015.

Mandzjuk Denys, " «Aktory prohraly, bo zabuly vzjaty sufljera». Jak halyc'kyj futbol počynavsja z teatral'noï višalky », document en ligne, consulté le 11.07.2016<http:// ua.tribuna.com/tribuna/blogs/deathintenitive/817086.html>.

Mrozik Agnieszka, « «Komuniści (nie) mają ojczyzny...», Wanda Wasilewska jako polska (anty)bohaterka narodowa », Studia Litteraria et Historica, 2013, n 2, pp. 528-554.

Navrockij Zigismund, Sem ’ja Januš [film], Minsk : Sovetskaja Belarus', 1941.

Navrockij Zigismund, Zigmund Kolosovskij [film], Kiev : Kievskaja kinostudija, 1945.

Oleg Korvjakov, Evgenij Gabrilovič. Poslednij avtograf [cycle de quatre films], Moscou : studija fondovyh i arhivnyh programm « Kul'tura », 1998.

Peterburgskij Nekropol', « Dmohovskij Boris Mihajlovič », 2013, document en ligne, consulté le 4.07.2016 <http://spb-tombs-walkeru.narod.ru/2013/6/19.htm>.

Pljatt Rostislav, Bez épiloga (red. Pojurovskij, B.M.), Moscou : Centrpoligraf, « Teatral'nye memuary $», 2000$.

Revuc'kyj Valerijan, «Vystavy za tvoramy i. franka na sceni ukraïns'koï dijaspory », Visnyk L'vivs'koho Universytetu, Serija Mystectvo, 2006, n 6. pp. 199-200.

Rittersporn Gábor T., Anguish, Anger, and Folkways in Soviet Russia, Pittsburgh : University of Pittsburgh Press, 2014.

Romm Mihail, Mečta [film], Moscou : Mosfil'm, 1941.

Romm Mihail, Izbrannye proizvedenija v 3-h tomah. T. 2 : O sebe, o ljudjah, o fil'mah, Moscou : Iskusstvo, 1981 (consulté en fichier électronique fb2).

Room Abram, Veter s Vostoka [film], Kiev : Kievskaja kinostudija, 1940.

Savčenko Viktor, Bogdan Hmelnickij [film], Kiev : Kievskaja kinostudija, 1941.

Shore Marci, Caviar and Ashes, A Warsaw Generation's Life and Death in Marxism, 19181968, New Haven \& London: Yale University Press, 2006.

Sorokin Wladimir, « Die Ukraine ist in uns eingedrungen », Frankfurter Allgemeine Zeitung, 21.07.2014, document en ligne traduit en russe, consulté le 21.06.2016 <http://rusorokin.livejournal.com/314777.html>.

Statystyka Polski, Drugi powszechny spis ludności z dn. 9 XII 1931 r., Warszawa : Adem Głównego Urządu Statystycznego, 1937-1938 (fasc. « Województwo Lwówskie bez miasta Lwówa »; « Miasto Lwów »). 
Symonenko Rem, « Vasylevs'kyj Leon », Encyklopedija istoriï Ukraïny, t. 1, Redkol.: V. A. Smolij (golova) ta in. NAN Ukraïny. Instytut istoriï Ukraïny, Kiev : Naukova dumka, 2003.

[TES] Ternopil's 'kyj encyklopedyčnyj slovnyk u 4 t. (redkol. : H. Javors'kyj ta in.), Ternopil' : Vydavnyčo-poligrafičnyj kombinat «Zbruč», 2004-2010.

Tokarev Vassilij, «Stalin i Vanda Vasilevskaja : limitirovannyj dialog (1940) », Przeglad Rusycystyczny, 2007, n 1 (117), pp. 39-57.

Tokarev Vassilij, « Maršrutami «Adama Mickeviča» : meždu Kievom i Ašhabadom (19401942) », Valérie Pozner, Irina Tcherneva, Vanessa Voisin (dir.), Perežit'vojnu. Sovetskaja kinoindustrija 1939-1949, Moscou : ROSSPÈN, 2017, à paraître.

Usievič Elena, Vanda Vasilevskaja : Kritiko-biografičeskij očerk, Moscou : Sovetskij pisatel', 1954.

Velymčanycja Ol'ha, "Amvrosij Bučma : tvorča dolja «rozumnogo Arlekina» », Kinoteatr, $2009, \mathrm{n}^{\circ} 2$, document en ligne, consulté le 9.07.2016<http://ktm.ukma.edu.ua/show_ content.php?id=884>.

Vengerov Leonid, Vanda Vasilevskaja : kritiko-biografičeskij očerk, Moscou : Gos. izd-vo Hudožestvennoj literatury, 1955.

Yekelchyk Serhy, Stalin's empire of memory : Russian-Ukrainian relations in the Soviet historical imagination, Toronto : University of Toronto press, cop. 2004. 


\section{Table des illustrations}

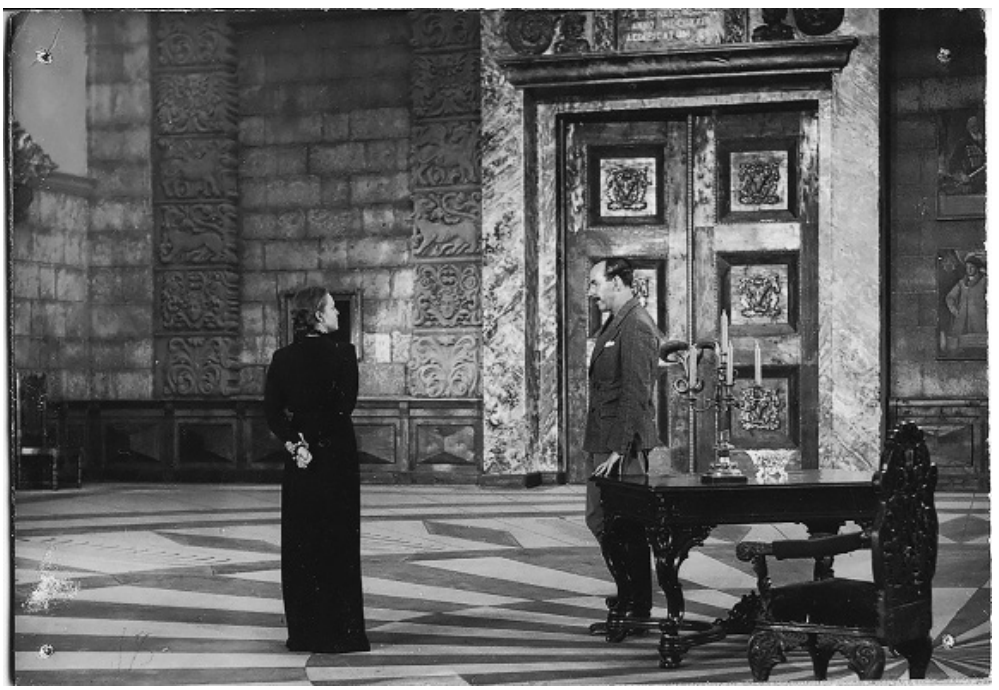

Fig. 01 : Chez la comtesse Przeżyńska (Vent d'Est - Nacional'na kinostudija hudožnih fil'miv imeni Oleksandra Dovženka - Muzej).

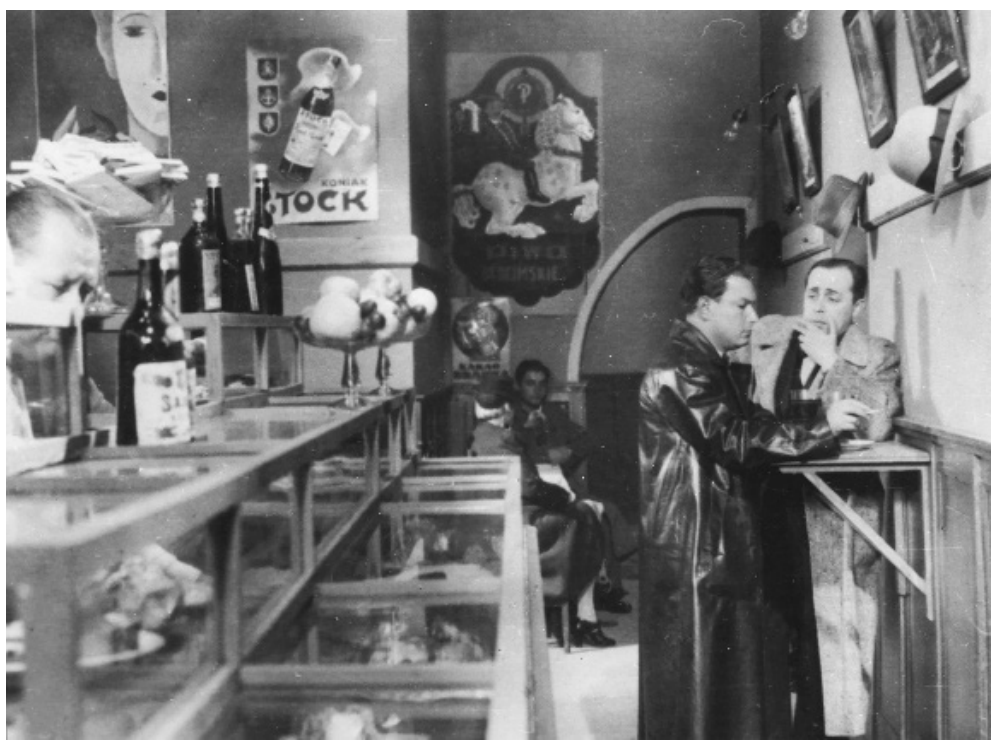

Fig. 02 : Un café polonais (Vent d'Est - Nacional'na kinostudija hudožnih fil'miv imeni Oleksandra Dovženka - Muzej). 


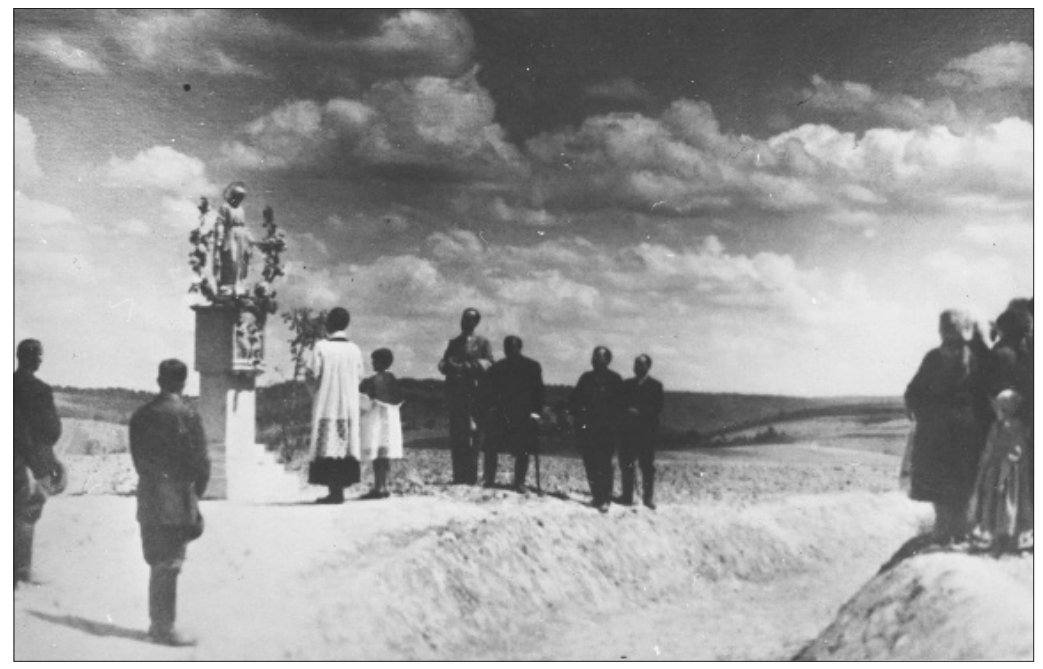

Fig. 03 : Cérémonie catholique (Vent d'Est - Nacional'na kinostudija hudožnih fil'miv imeni Oleksandra Dovženka - Muzej).

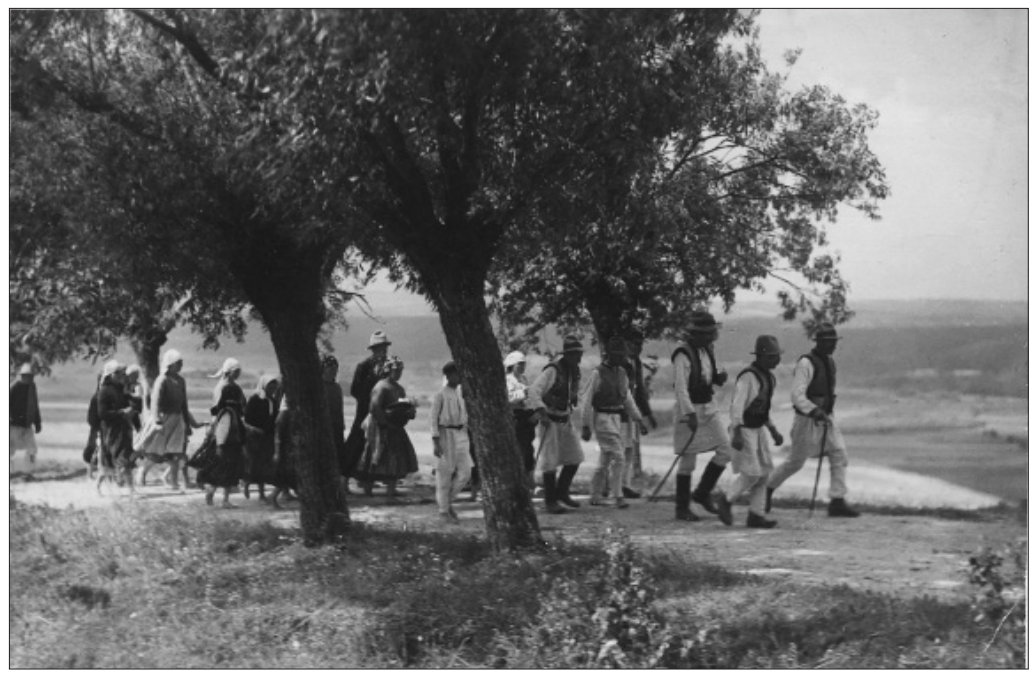

Fig. 04 : Paysans ukrainiens (Vent d'Est - Nacional'na kinostudija hudožnih fil'miv imeni Oleksandra Dovženka - Muzej). 


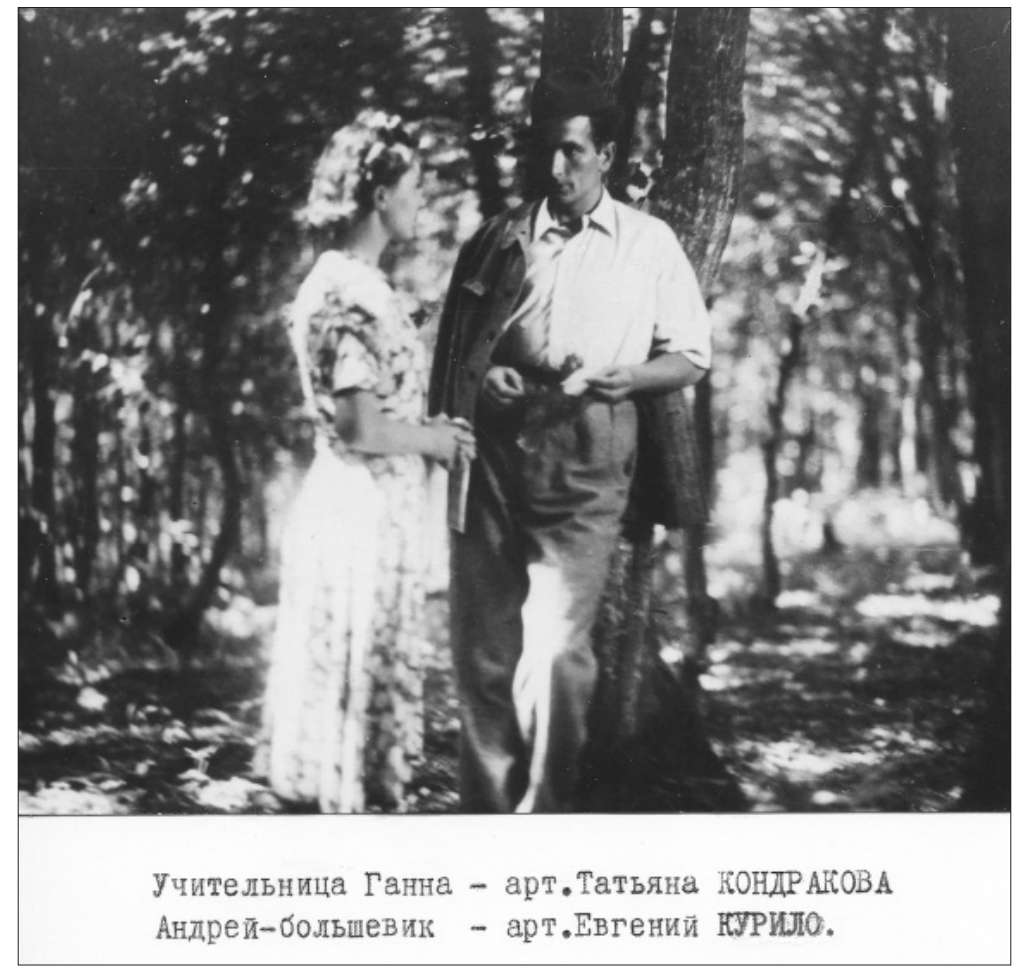

Fig. 05 : L'institutrice Ganna et André-le-bolchevik (Vent d'Est - Nacional'na kinostudija hudožnih fil'miv imeni Oleksandra Dovženka - Muzej).

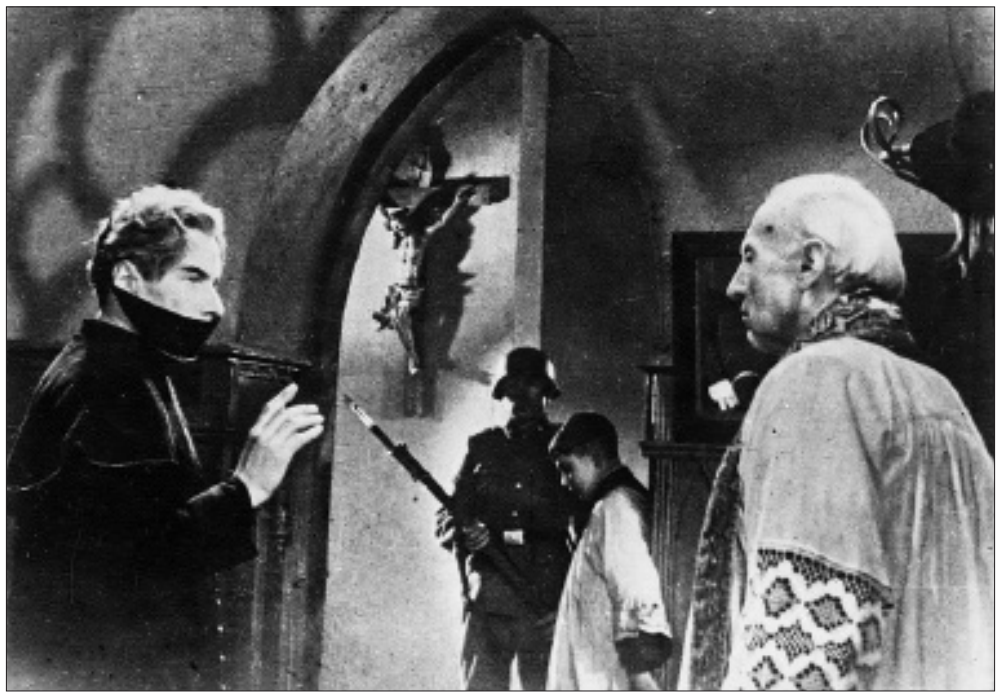

Fig. 06 : Zigmund Kolosovski (Nacional'na kinostudija hudožnih fil'miv imeni Oleksandra Dovženka - Muzej). 


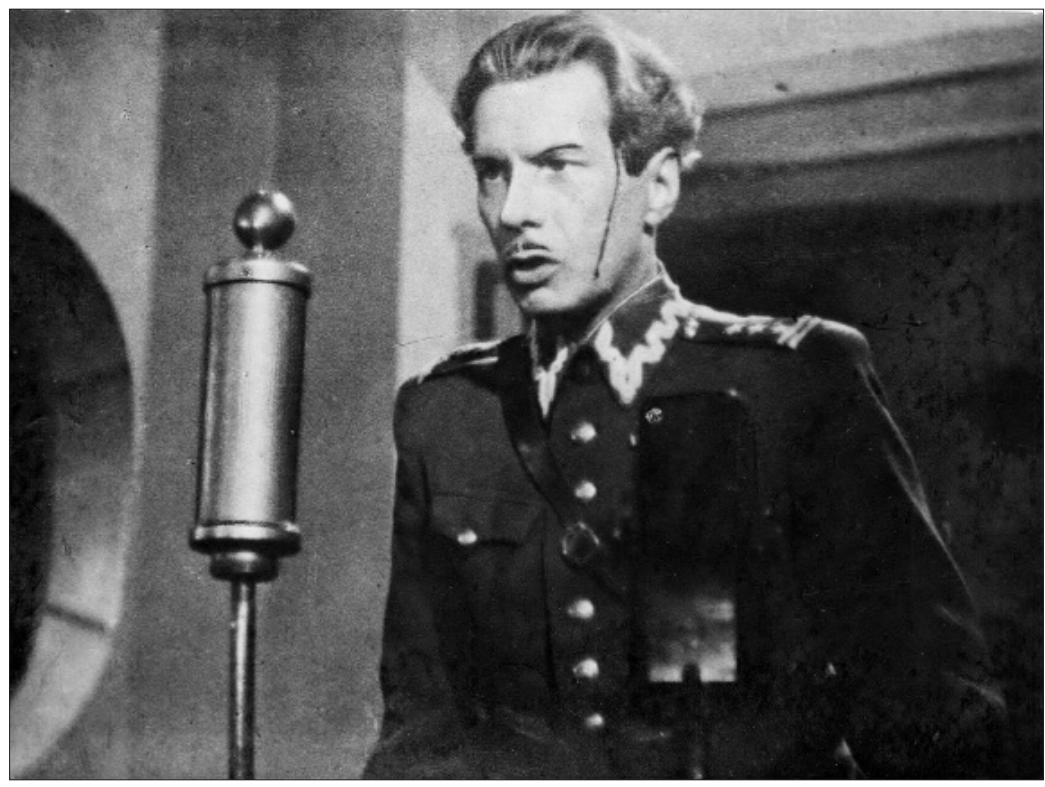

Fig. 07 : Zigmund Kolosovski (Nacional'na kinostudija hudožnih fil'miv imeni Oleksandra Dovženka - Muzej).

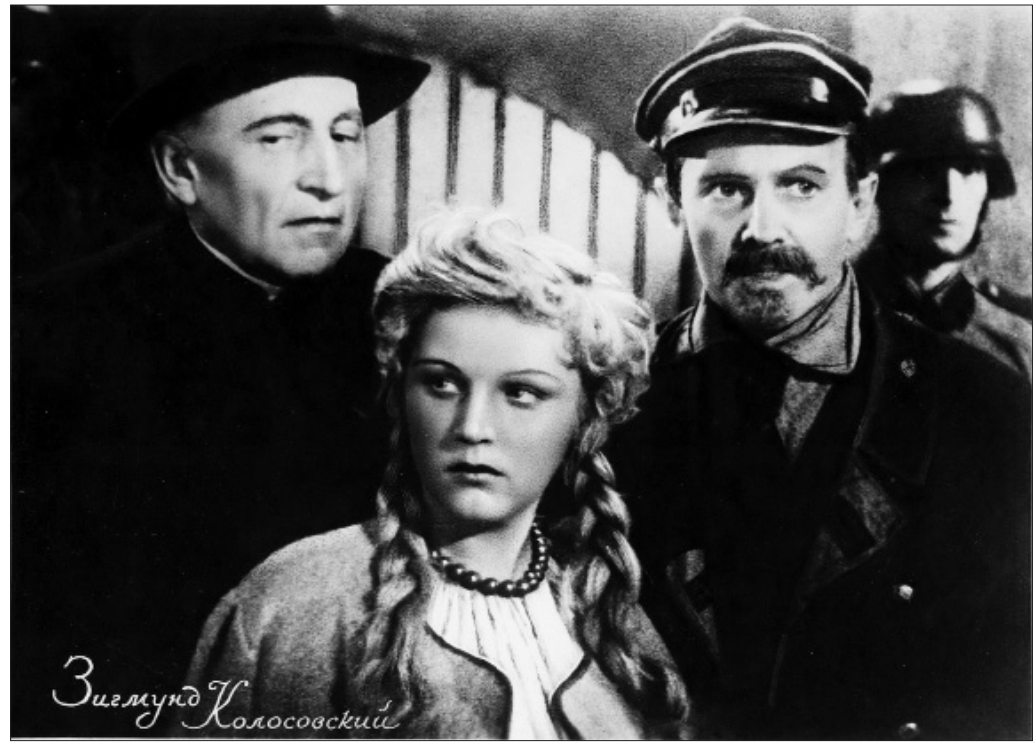

Fig. 08 : Zigmund Kolosovski (Nacional'na kinostudija hudožnih fil'miv imeni Oleksandra Dovženka - Muzej). 


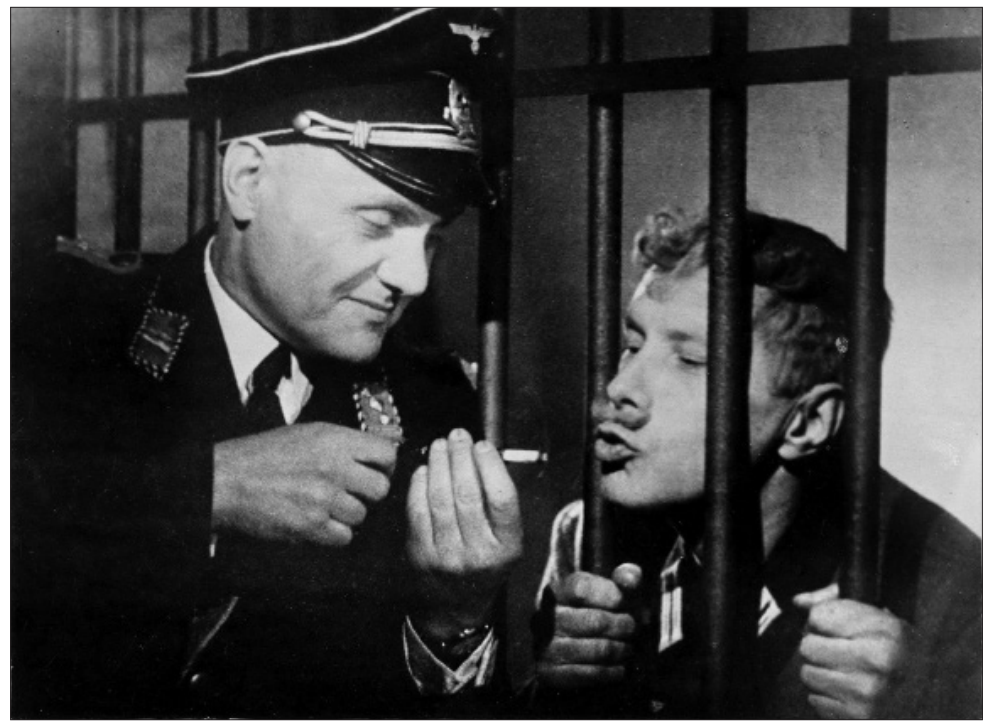

Fig. 09 : Zigmund Kolosovski (Nacional'na kinostudija hudožnih fil'miv imeni Oleksandra Dovženka - Muzej).

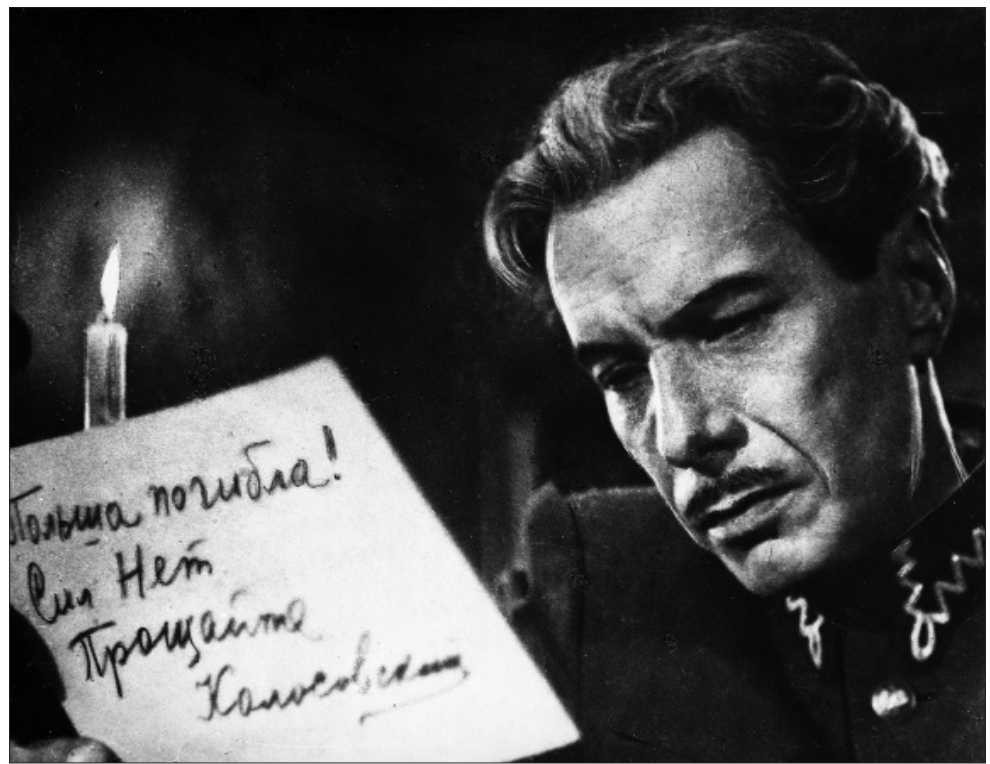

Fig. 10 : Boris Dmokhovski dans le rôle de Zigmund Kolosovski (Nacional'na kinostudija hudožnih fil'miv imeni Oleksandra Dovženka - Muzej). 


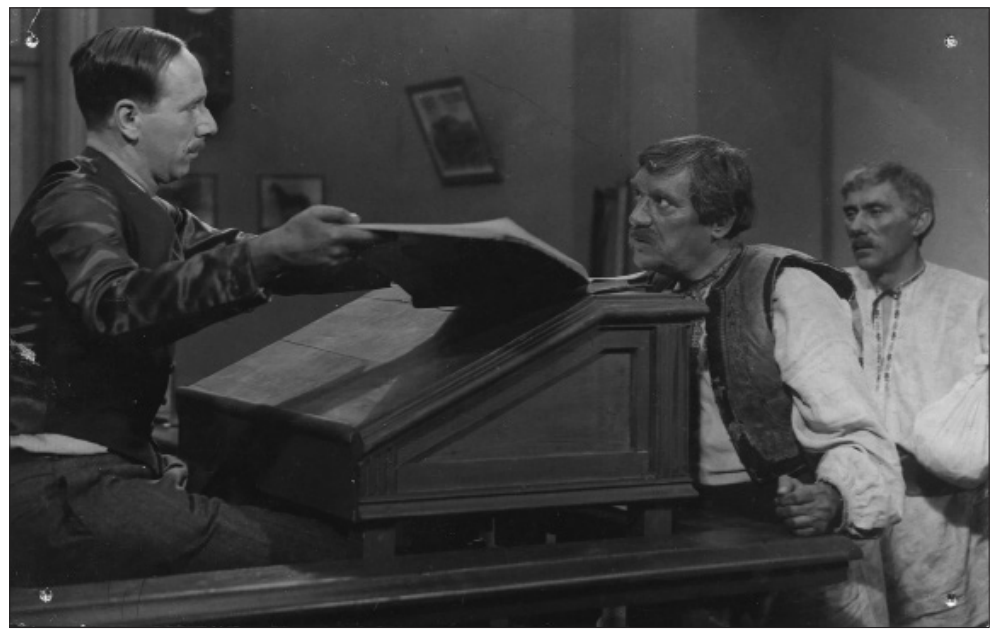

Fig. 11 : Rostislav Pliatt face à Amvrosi Butchma (Vent d'Est Nacional'na kinostudija hudožnih fil'miv imeni Oleksandra Dovženka - Muzej).

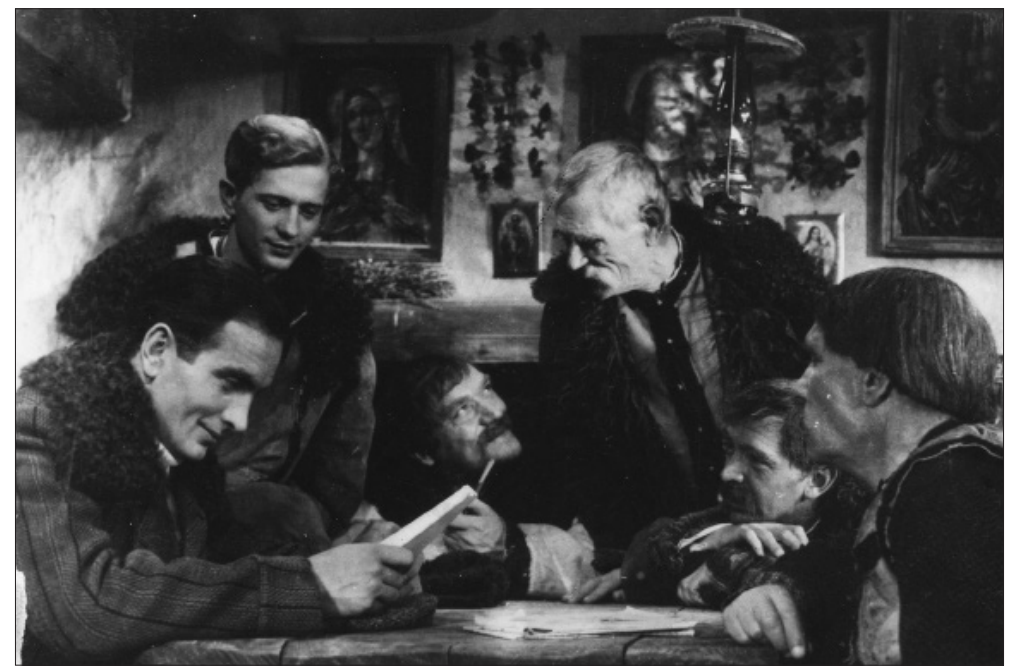

Fig. 12 : Andriï Kurilo et Orest Slipenky (les deux plus jeunes à gauche), Petro Soroka (assis, la tête sur les mains, avant dernier à droite) dans Vent d'Est (Nacional'na kinostudija hudožnih fil'miv imeni Oleksandra Dovženka - Muzej). 


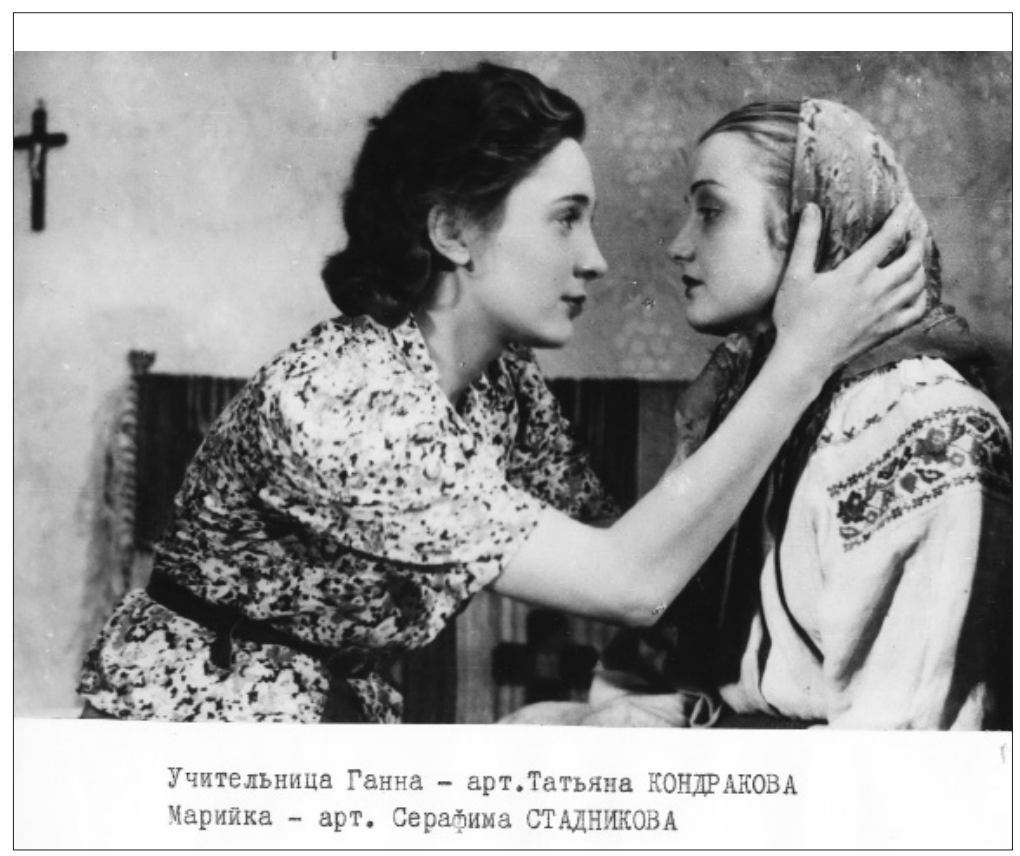

Fig. 13 : Stefa Stadnykivna (à droite) dans Vent d'Est

(Nacional'na kinostudija hudožnih fil'miv imeni

Oleksandra Dovženka - Muzej).

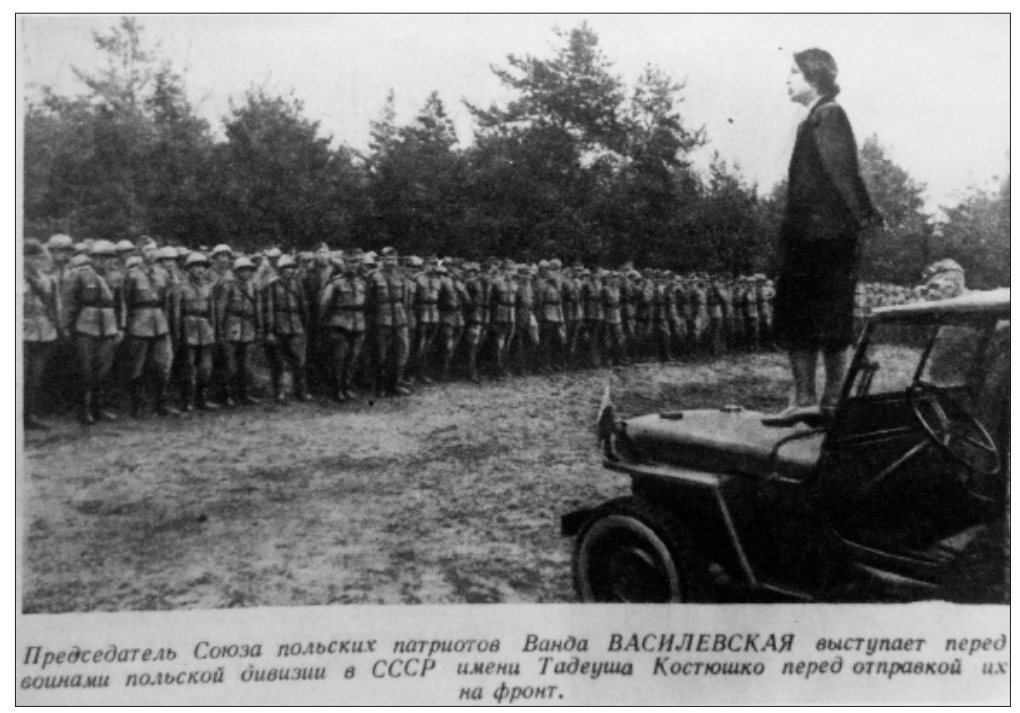

Fig. 14 : Wanda Wasilewska harangue les soldats de de la division Kościuszko qui combat avec l'Armée rouge (CDAMLM, fond 73, op. 1, d. 834). 
La Pologne, un sujet soviétique? ...

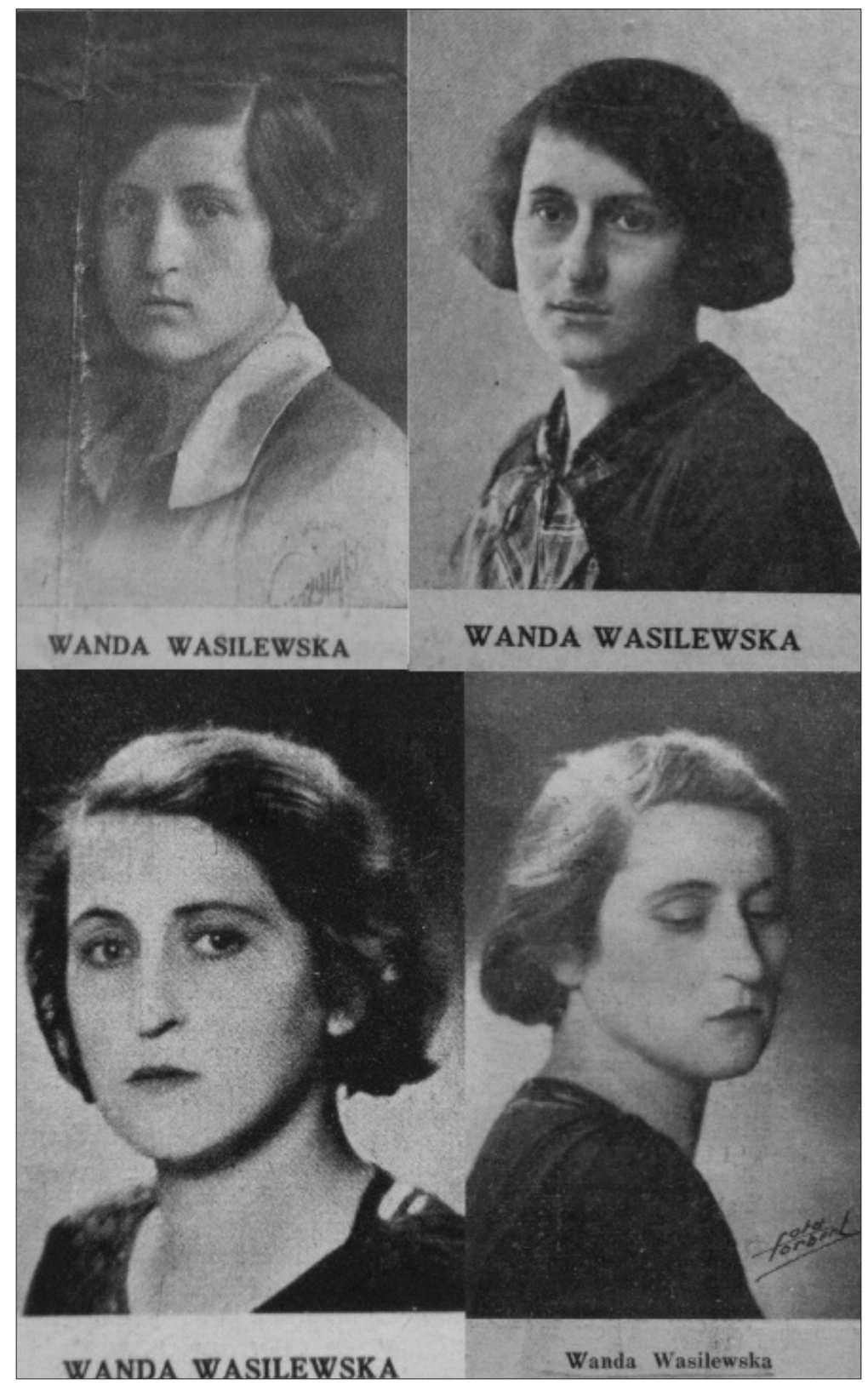

Fig. 15 : Wanda Wasilewska dans la presse polonaise entre 1934 et 1938 (CDAMLM, fond 73, op. 1, d. 761). 
Éric AunOBLe

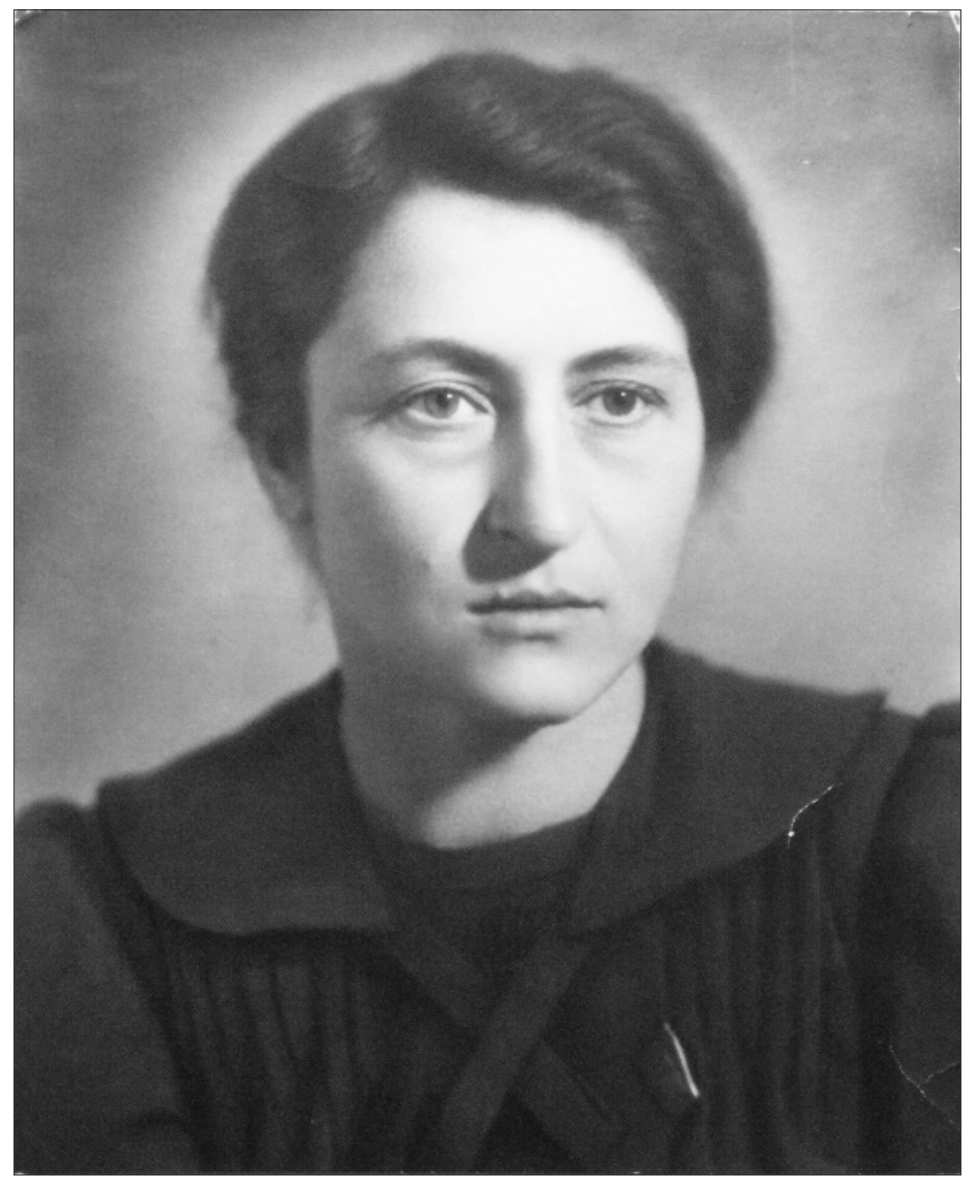

Fig. 16 : Wanda Wasilewska députée au Soviet suprême en 1940 (CDAMLM, fond 73, op. 1, d. 834). 
La Pologne, un sujet soviétique ? ...

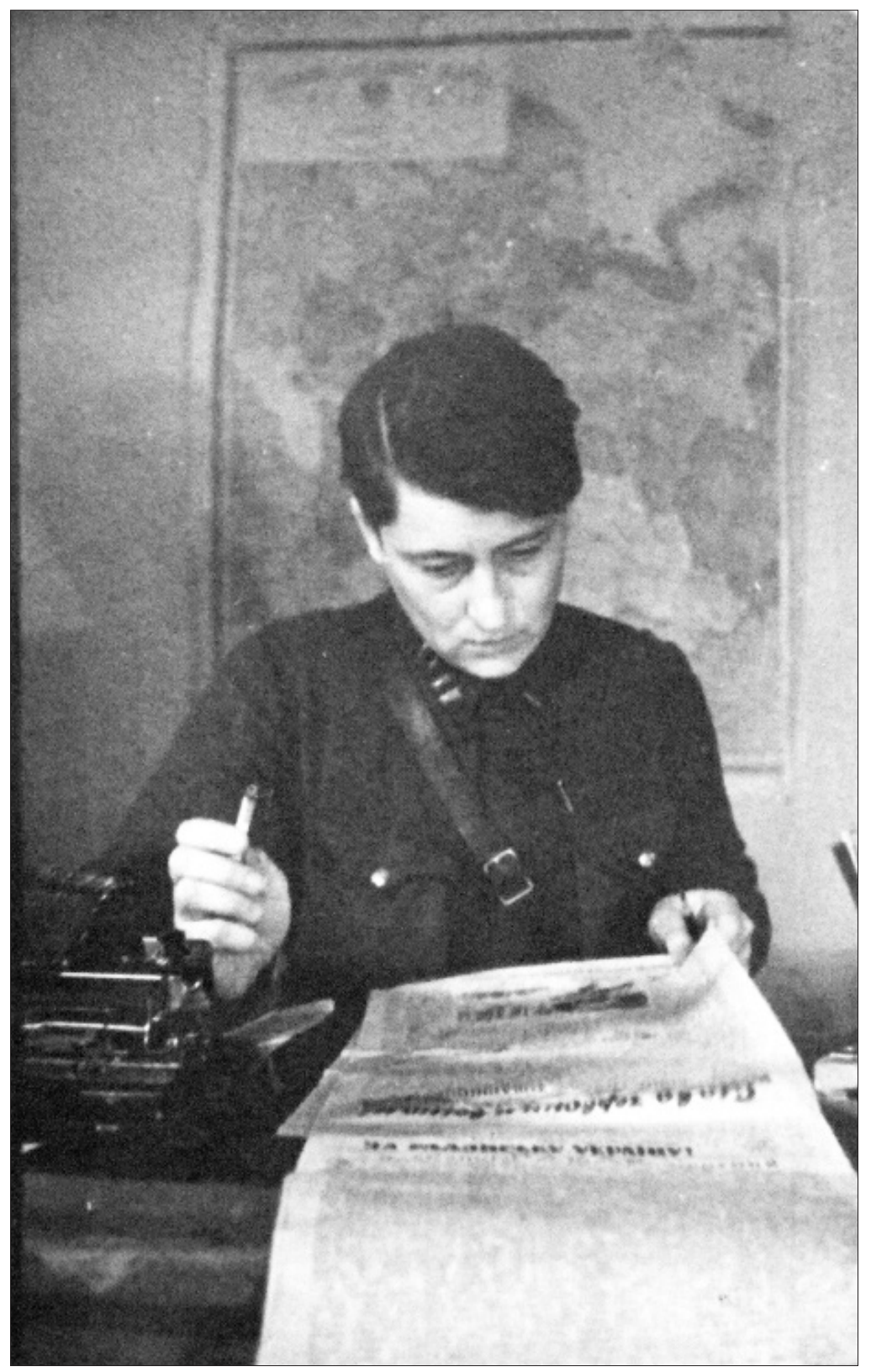

Fig. 17 : Wanda Wasilewska, cadre politique dans l'Armée rouge (c. 1941-1942 - CDAMLM, fond 73, op. 1, d. 834). 


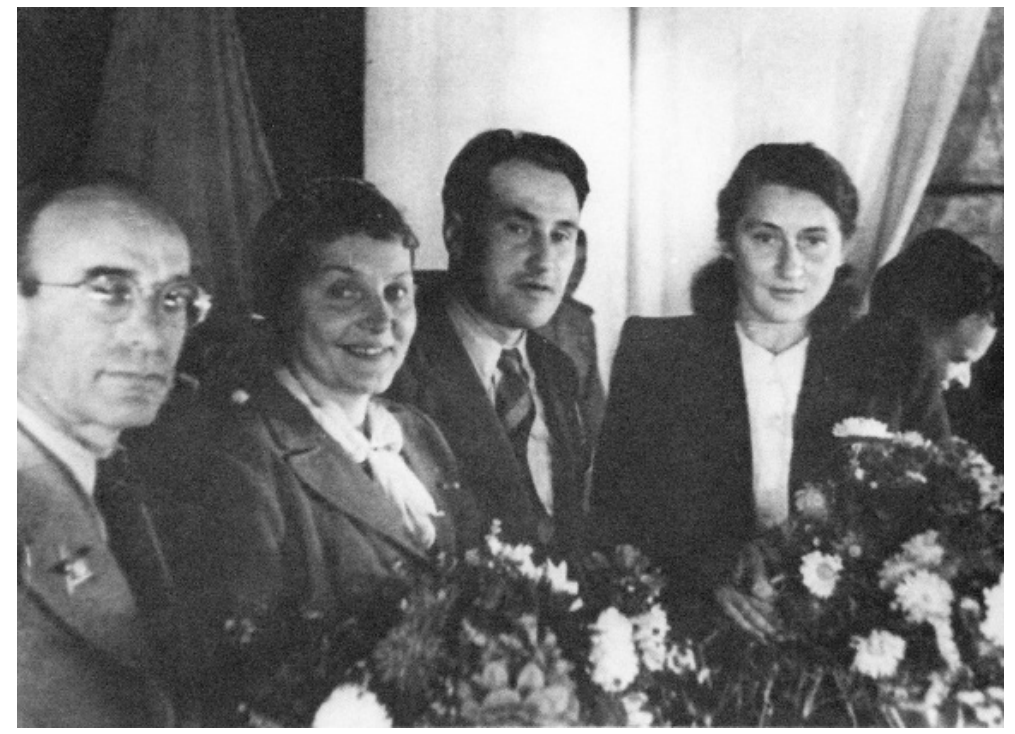

Fig. 18 : L'équipe du film Arc en Ciel : Oskar Sandler (compositeur), Natalia Ujviï (actrice), Mark Donskoï (réalisateur), Wanda Wasilewska (sénariste) (Nacional'na kinostudija hudožnih fil'miv imeni Oleksandra Dovženka - Muzej). 